\title{
Kernos
}

Revue internationale et pluridisciplinaire de religion grecque antique

30 | 2017

Varia

\section{Le règlement religieux de Marmarini (Thessalie) : nouvelles lectures, nouvelles interprétations}

\section{Richard Bouchon et Jean-Claude Decourt}

\section{OpenEdition}

Journals

Édition électronique

URL : http://journals.openedition.org/kernos/2494

DOI : 10.4000/kernos.2494

ISSN : 2034-7871

Éditeur

Centre international d'étude de la religion grecque antique

Édition imprimée

Date de publication : 1 octobre 2017

Pagination : 159-186

ISSN : 0776-3824

Référence électronique

Richard Bouchon et Jean-Claude Decourt, « Le règlement religieux de Marmarini (Thessalie) :

nouvelles lectures, nouvelles interprétations », Kernos [En ligne], 30 | 2017, mis en ligne le 01 octobre

2019, consulté le 18 septembre 2020. URL : http://journals.openedition.org/kernos/2494 ; DOI :

https://doi.org/10.4000/kernos.2494

Ce document a été généré automatiquement le 18 septembre 2020

Kernos 


\title{
Le règlement religieux de Marmarini (Thessalie) : nouvelles lectures, nouvelles interprétations
}

\author{
Richard Bouchon et Jean-Claude Decourt
}

1 L'importance de la longue inscription opisthographe portant un règlement religieux trouvée il y a quelques années à Marmarini, sur le territoire de l'antique Larissa, et publiée dans cette même revue par J.-C. Decourt et A. Tziafalias en $2015^{1}$, a déjà été soulignée par les commentateurs, R. Parker et S. Scullion la qualifiant de «bombshell $»^{2}$. Les remarques faites par ces commentateurs, la rédaction des notices du Bulletin épigraphique ${ }^{3}$, la consultation de nouvelles photographies ainsi qu'un patient travail sur les estampages en dépôt à la Maison de l'Orient à Lyon par R. Bouchon nous conduisent à proposer aujourd'hui une nouvelle édition et une nouvelle traduction de ce texte. Elles sont suivies d'un commentaire qui ne se veut pas exhaustif, mais qui se limite à présenter les corrections et les nouveautés les plus importantes ${ }^{4}$. Pour des raisons que nous développons au début de notre commentaire et pour éviter les confusions, nous nommons désormais la face la mieux conservée (Face B de Decourt et Tziafalias) Face I et celle qui est en moins bon état (Face A de Decourt et Tziafalias) Face II.

\section{Texte}

\section{Face I}

\begin{tabular}{|c|c|}
\hline & 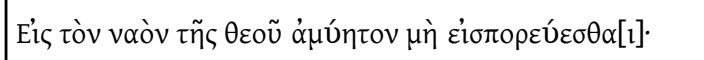 \\
\hline & 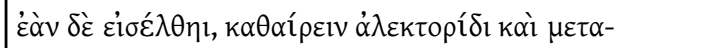 \\
\hline & 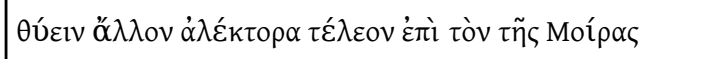 \\
\hline 4 & 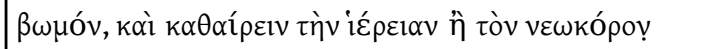 \\
\hline & 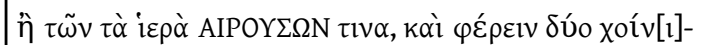 \\
\hline
\end{tabular}




\begin{tabular}{|c|c|}
\hline & 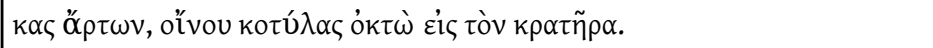 \\
\hline & 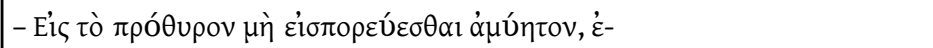 \\
\hline 8 & 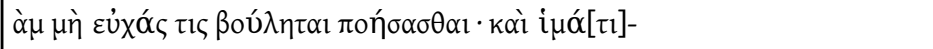 \\
\hline & 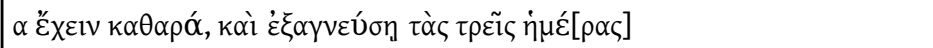 \\
\hline & 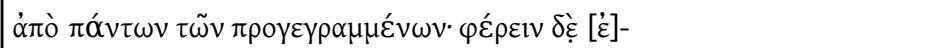 \\
\hline & 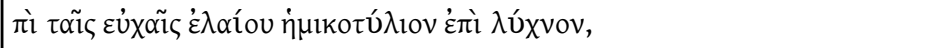 \\
\hline 12 & 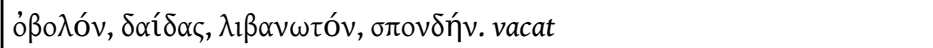 \\
\hline & 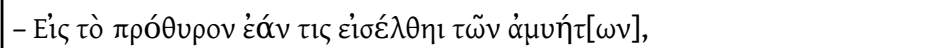 \\
\hline & 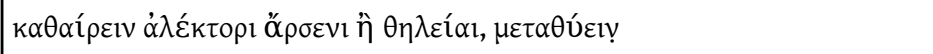 \\
\hline & 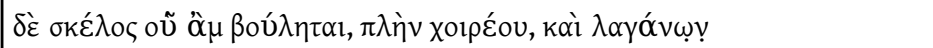 \\
\hline 16 & 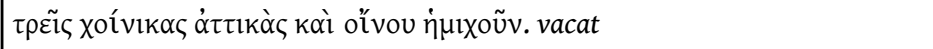 \\
\hline & 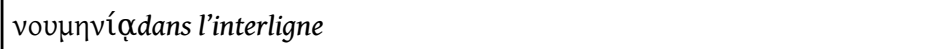 \\
\hline & 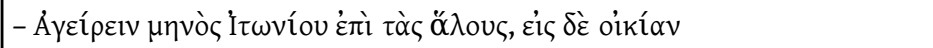 \\
\hline & 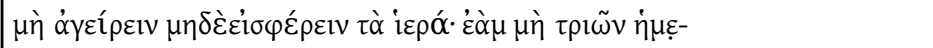 \\
\hline & 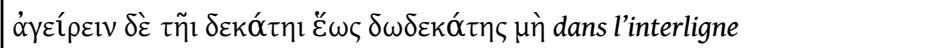 \\
\hline & 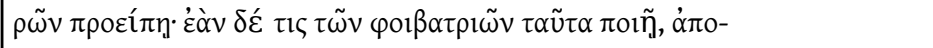 \\
\hline 20 & 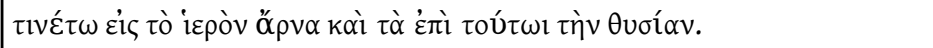 \\
\hline & 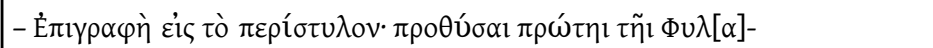 \\
\hline & 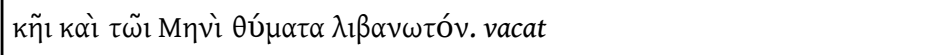 \\
\hline & 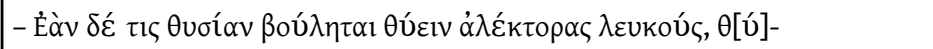 \\
\hline 24 & 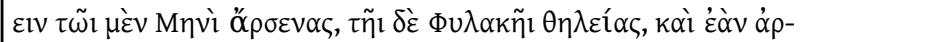 \\
\hline & 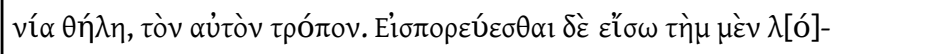 \\
\hline & 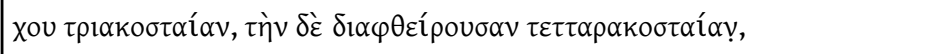 \\
\hline & 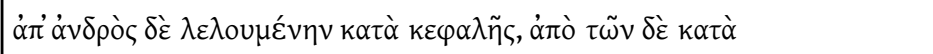 \\
\hline 28 & 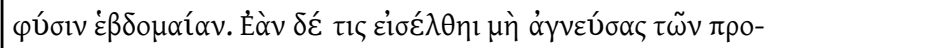 \\
\hline & 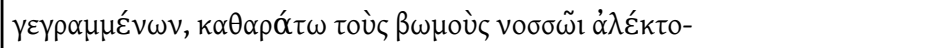 \\
\hline & 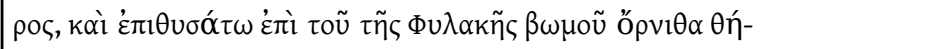 \\
\hline & 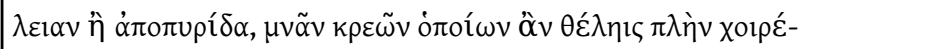 \\
\hline 32 & 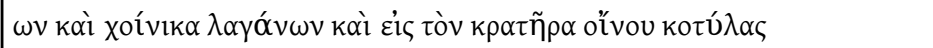 \\
\hline & 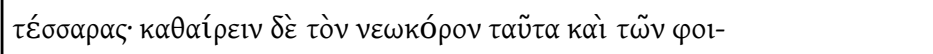 \\
\hline & 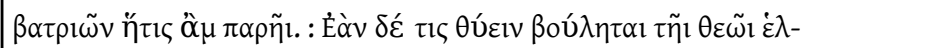 \\
\hline & 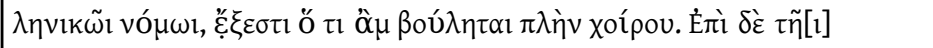 \\
\hline 36 & 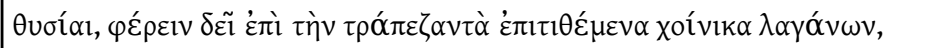 \\
\hline & 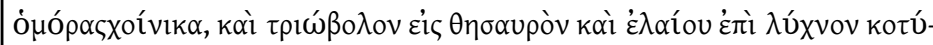 \\
\hline & 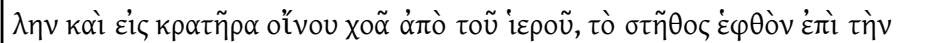 \\
\hline
\end{tabular}




\begin{tabular}{|c|c|}
\hline & 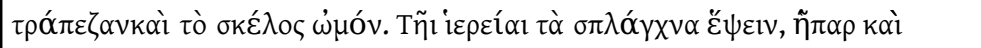 \\
\hline \multirow[t]{4}{*}{40} & 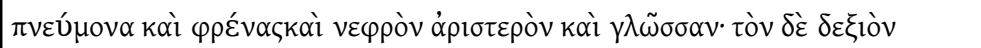 \\
\hline & 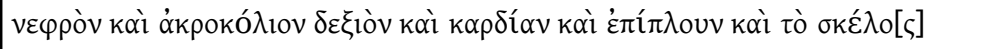 \\
\hline & 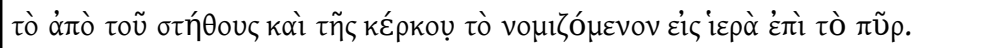 \\
\hline & 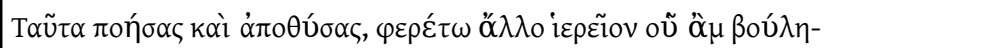 \\
\hline \multirow[t]{4}{*}{44} & 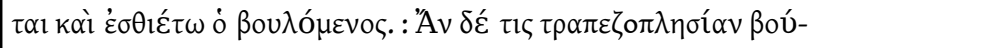 \\
\hline & 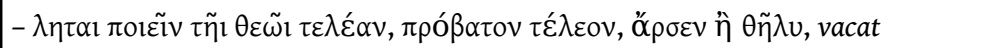 \\
\hline & 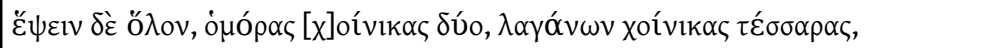 \\
\hline & 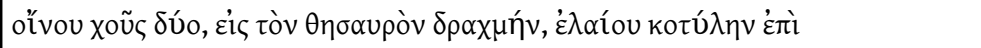 \\
\hline \multirow[t]{4}{*}{48} & 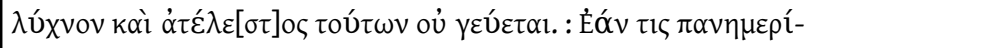 \\
\hline & 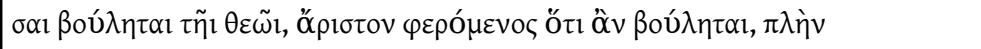 \\
\hline & 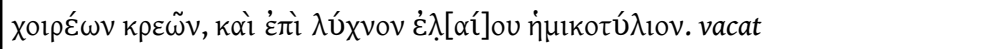 \\
\hline & 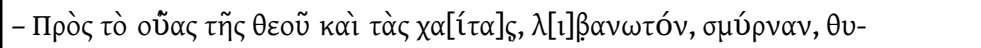 \\
\hline \multirow[t]{4}{*}{52} & 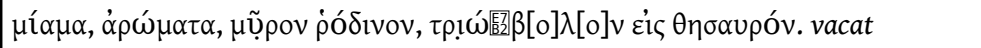 \\
\hline & 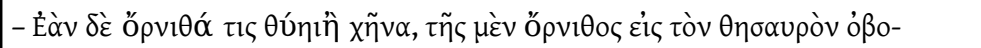 \\
\hline & 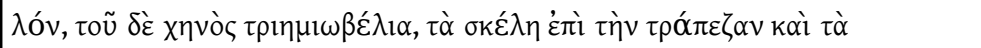 \\
\hline & 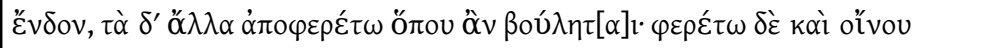 \\
\hline \multirow[t]{4}{*}{56} & 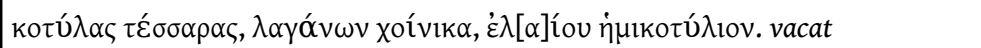 \\
\hline & 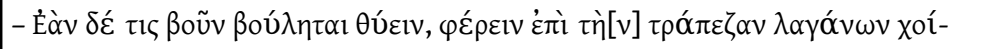 \\
\hline & 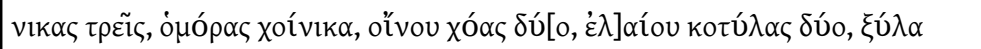 \\
\hline & 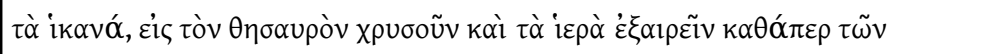 \\
\hline \multirow[t]{4}{*}{60} & 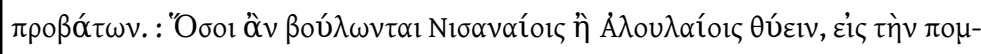 \\
\hline & 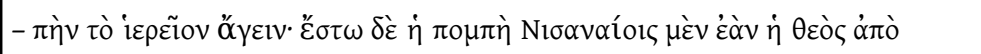 \\
\hline & 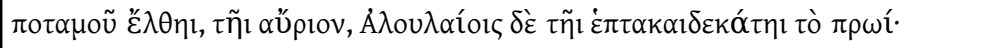 \\
\hline & 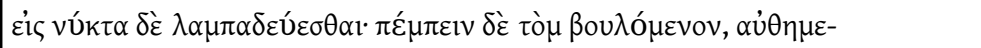 \\
\hline \multirow[t]{4}{*}{64} & 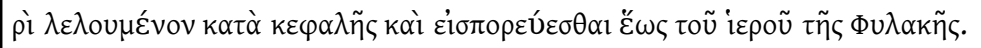 \\
\hline & 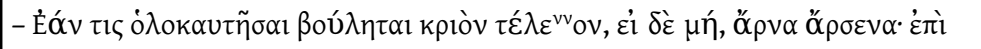 \\
\hline & 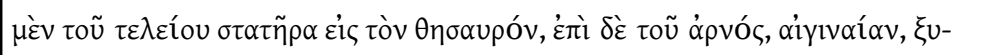 \\
\hline & 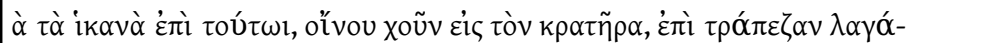 \\
\hline \multirow[t]{4}{*}{68} & 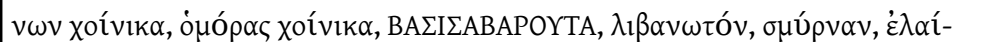 \\
\hline & 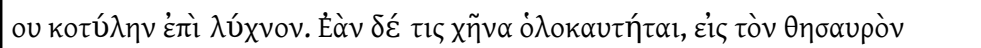 \\
\hline & 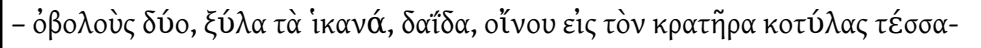 \\
\hline & 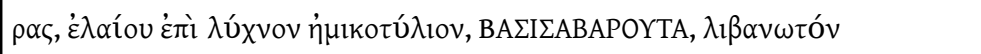 \\
\hline \multirow[t]{2}{*}{72} & 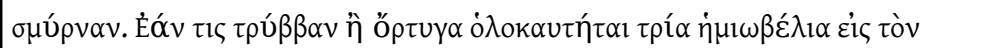 \\
\hline & 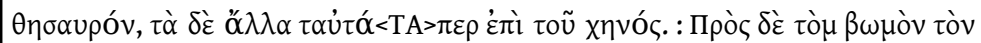 \\
\hline
\end{tabular}




\begin{tabular}{|c|c|}
\hline & 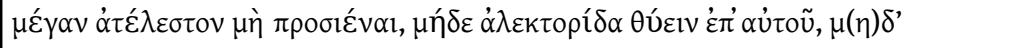 \\
\hline & 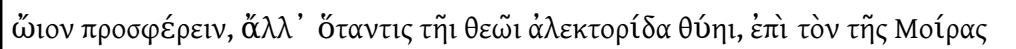 \\
\hline \multirow[t]{4}{*}{76} & 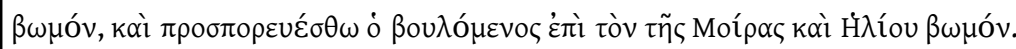 \\
\hline & 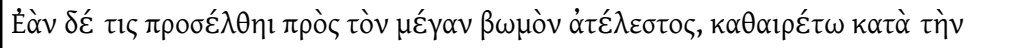 \\
\hline & 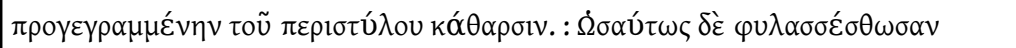 \\
\hline & 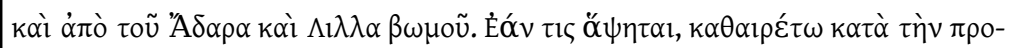 \\
\hline \multirow[t]{2}{*}{80} & 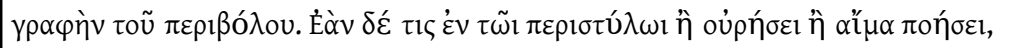 \\
\hline & 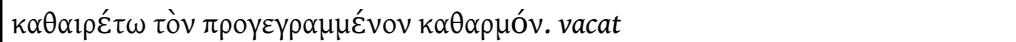 \\
\hline
\end{tabular}

\section{Face II}

\begin{tabular}{|c|c|}
\hline & - - - - - - - - peut-être traces de lettres (voir commentaire) - - \\
\hline & 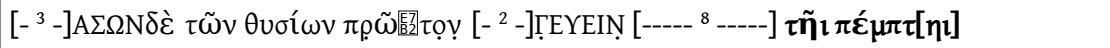 \\
\hline & 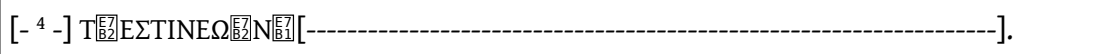 \\
\hline & 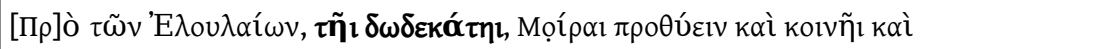 \\
\hline 4 & 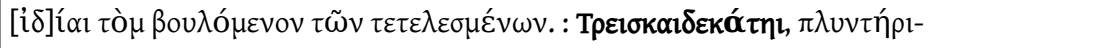 \\
\hline & 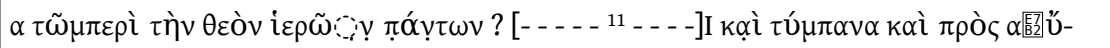 \\
\hline & 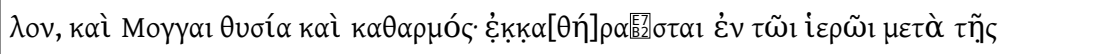 \\
\hline & 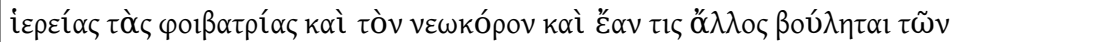 \\
\hline 8 & 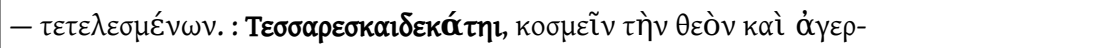 \\
\hline & 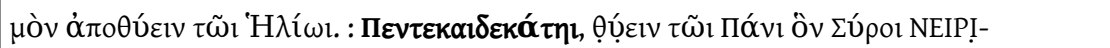 \\
\hline & 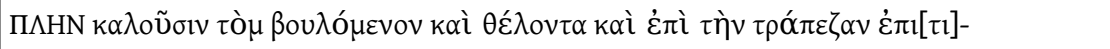 \\
\hline & 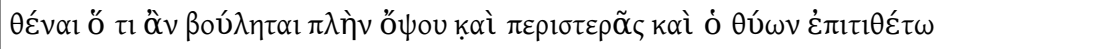 \\
\hline 12 & 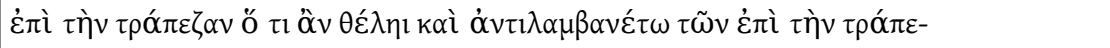 \\
\hline & 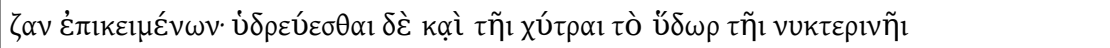 \\
\hline & 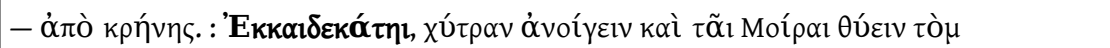 \\
\hline & 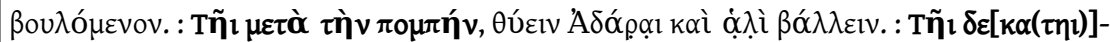 \\
\hline 16 & 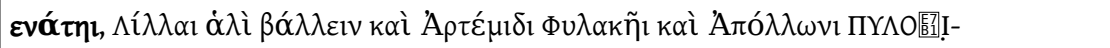 \\
\hline & 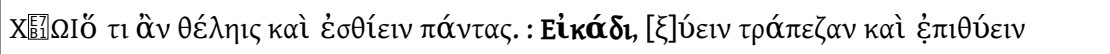 \\
\hline & 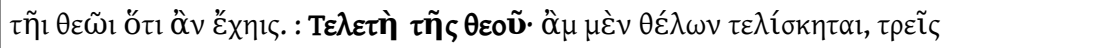 \\
\hline & 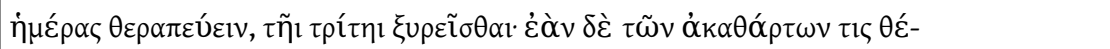 \\
\hline 20 & 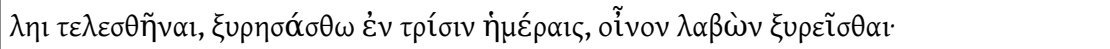 \\
\hline & 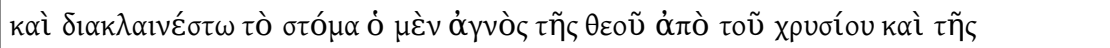 \\
\hline & 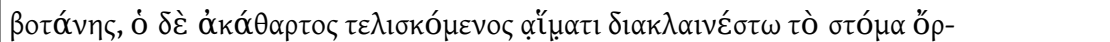 \\
\hline & 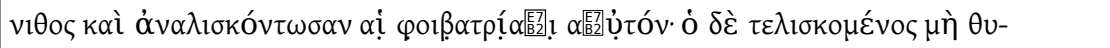 \\
\hline
\end{tabular}




\begin{tabular}{|c|c|}
\hline \multirow[t]{4}{*}{24} & 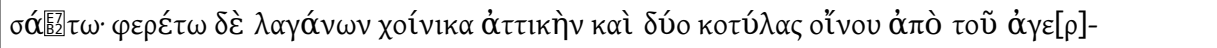 \\
\hline & 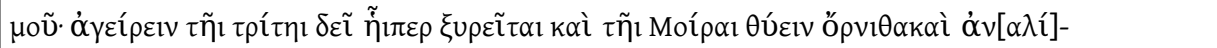 \\
\hline & 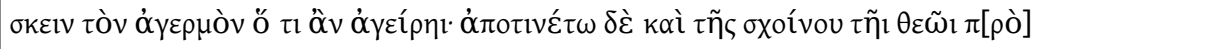 \\
\hline & 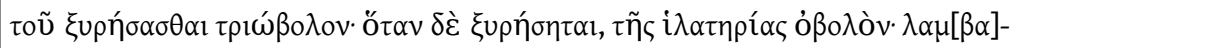 \\
\hline \multirow[t]{4}{*}{28} & 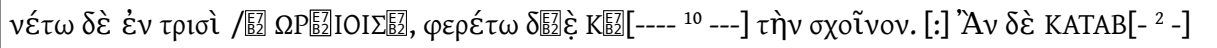 \\
\hline & 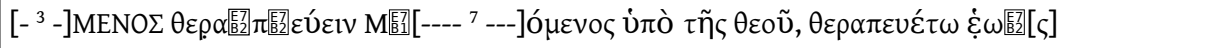 \\
\hline & 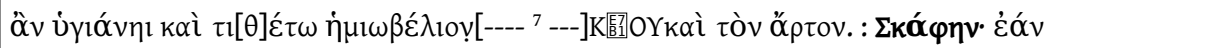 \\
\hline & 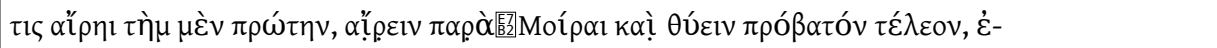 \\
\hline \multirow[t]{4}{*}{32} & 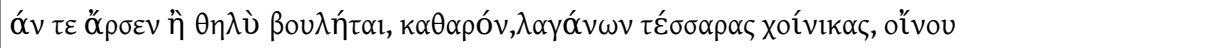 \\
\hline & 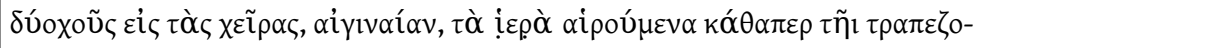 \\
\hline & 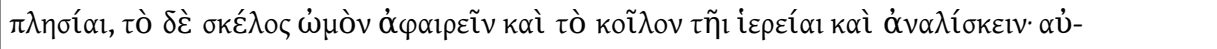 \\
\hline & 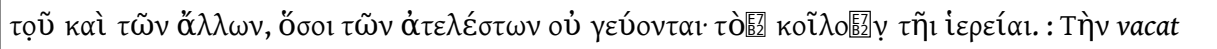 \\
\hline \multirow[t]{4}{*}{36} & 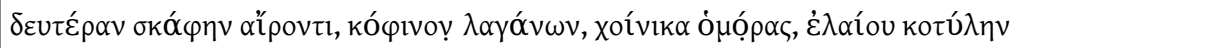 \\
\hline & 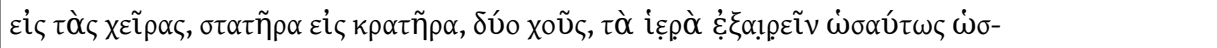 \\
\hline & 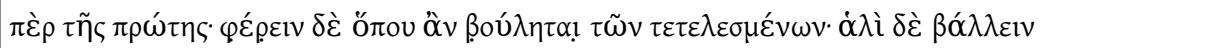 \\
\hline & 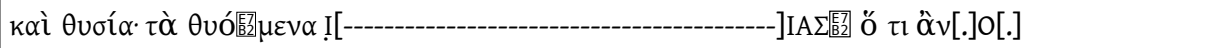 \\
\hline \multirow[t]{4}{*}{40} & 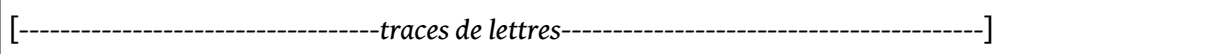 \\
\hline & 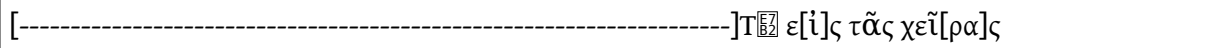 \\
\hline & 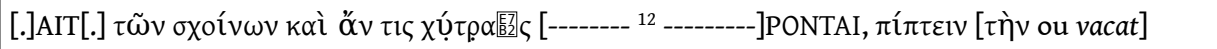 \\
\hline & 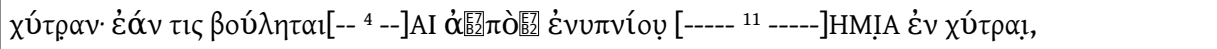 \\
\hline \multirow[t]{4}{*}{44} & 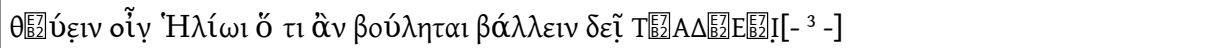 \\
\hline & 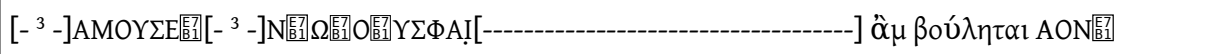 \\
\hline & 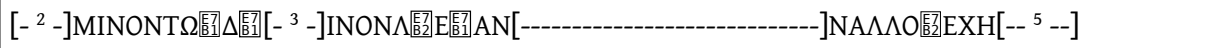 \\
\hline & 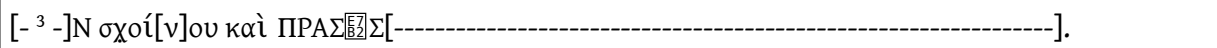 \\
\hline \multirow[t]{4}{*}{48} & 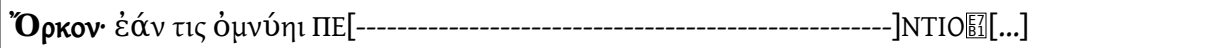 \\
\hline & 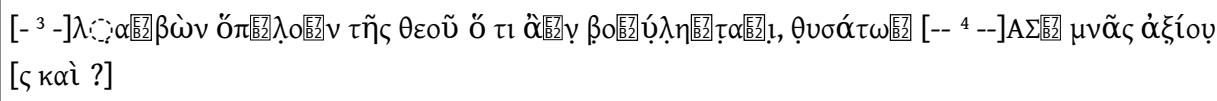 \\
\hline & 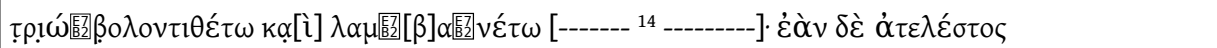 \\
\hline & 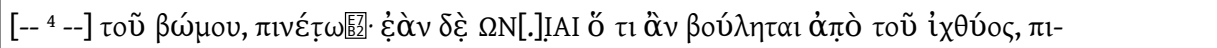 \\
\hline \multirow[t]{4}{*}{52} & y \\
\hline & 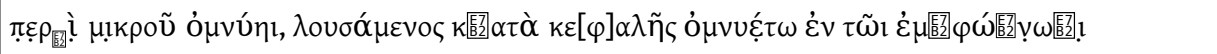 \\
\hline & 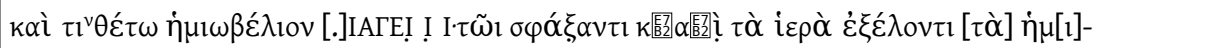 \\
\hline & $\omega \beta \varepsilon ́ \lambda ı \alpha$. \\
\hline
\end{tabular}




\section{Notes critiques}

2 Dans les notes qui suivent, BE renvoie au Bulletin épigraphique (2016), DT à DECOURT TZIAFALIAS (2015), C à CARBON (2016), et PS à PARKER - SCULLION (2016). Nous avons conservé dans la traduction les paragraphoi du texte grec.

\section{Face I}

L'inscription commence $7 \mathrm{~cm}$ sous la moulure de la stèle. 1. 4. On distingue la partie

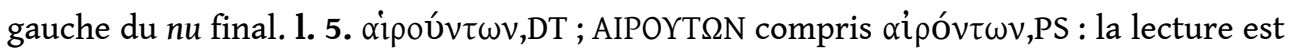

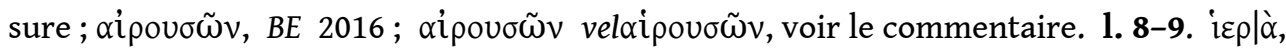

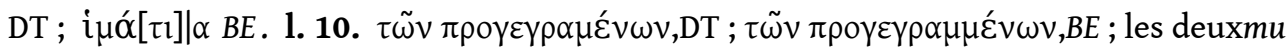

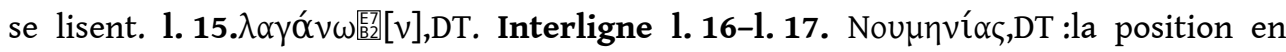

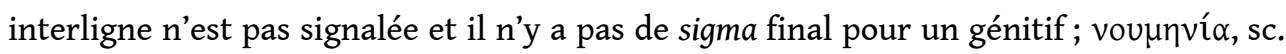

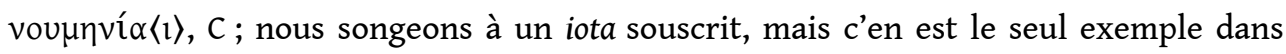

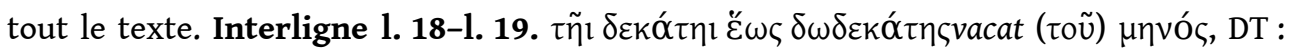

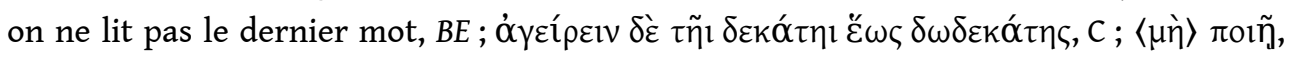

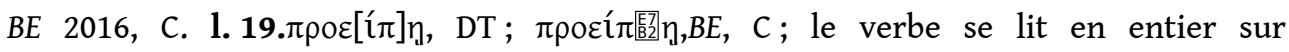

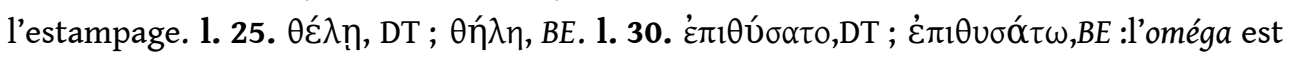

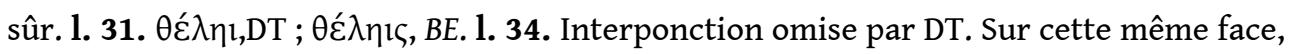
en ajouter aussi 1. 44, 48, 60 et 78. 1. 35. ô ò $\mu, \mathrm{DT}$, qui oublient $\tau$ bien lisible ; ǒ $\tau \imath \grave{\alpha} \mu, B E$.

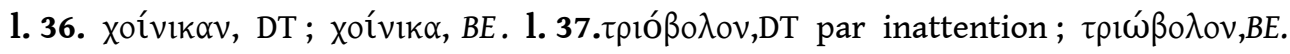

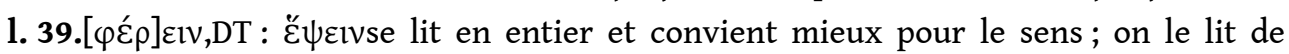

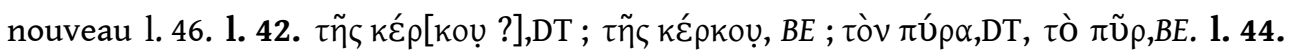

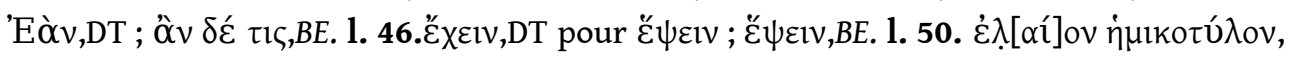

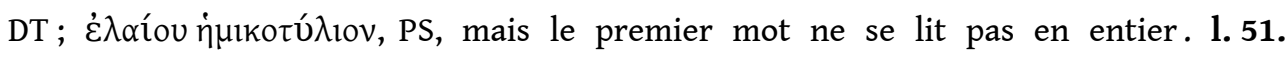
$\zeta \mu u ́ \rho v \alpha[v], D T ; \sigma \mu u ́ \rho v \alpha v, B E$;le sigma est assuré, comme 1.68 et 72. 1. 51/52. $\theta u \mid \mu i ́ \alpha v, D T$; $\theta v \mu \imath \tilde{\alpha} v$, PS qui y lisent un infinitif d'ordre; $\theta v \mid \mu i \alpha \mu \alpha, B E ; \tau \rho[10] \beta\left[0 \lambda_{0}\right] v$, DT. 1. 53.

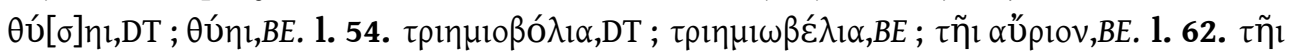

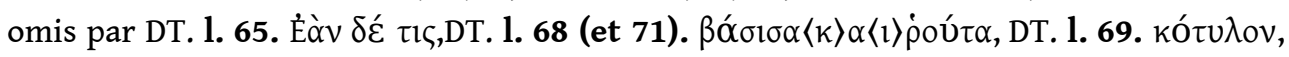

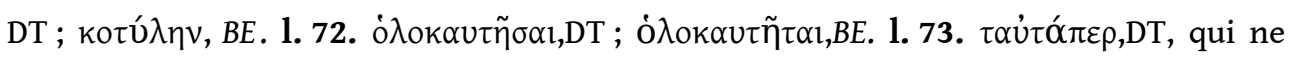

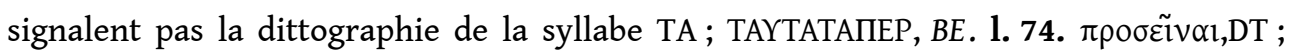

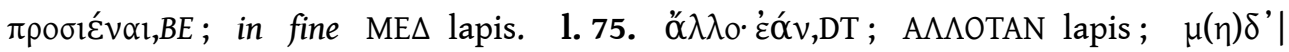

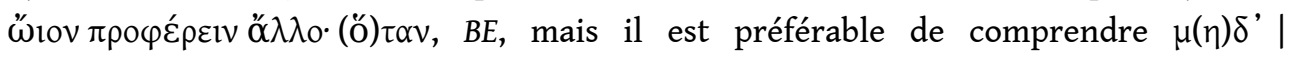

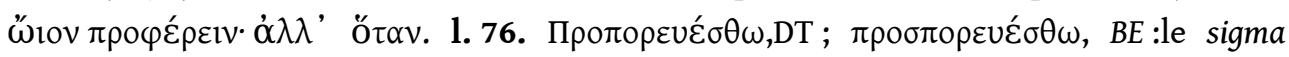
omis est bien lisible. 1. 79. $\alpha U ̛ \eta \tau \alpha, D T ; ~ \alpha \check{\psi} \psi \tau \tau \alpha 1, B E$.

\section{Face II}

Il manquerait au début 21 lignes, si l'on admet que le texte a été gravé immédiatement sous la moulure supérieure, ou 14 lignes, si on le fait débuter à la même hauteur que sur l'autre face. Nous sommes cependant d'avis que le haut de la stèle ne portait pas de texte gravé: voir le commentaire. 1. 1. AT $\Omega \mathrm{N}, \mathrm{DT}$; la lecture $-\mathrm{A} \Sigma \Omega \mathrm{N}$ - pourrait se restituer [.. $\dot{\alpha} \pi] \alpha \sigma \tilde{\omega} v$ ou $[\sigma u \mu \pi] \alpha \sigma \tilde{\omega} v$. La séquence -] ṬEYEIṆ[- n'a pas été lue par DT ; on

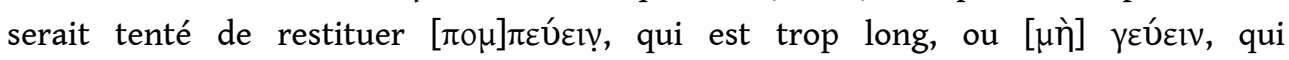
correspondrait mieux aux traces repérées; $\pi \varepsilon ́ \mu \pi \tau \eta ı$ sans l'article, DT. 1. 2. La lecture 
DT, à l'exception de la séquence TELTINE, est différente, mais ne se laisse pas mieux interpréter à droite, car trop lacunaire. 1. 3. [2-3 1.] A $\tau \tilde{\omega} \nu$ 'E $\lambda$ ov $\lambda \alpha i ́ \omega v, D T$; la lecture et

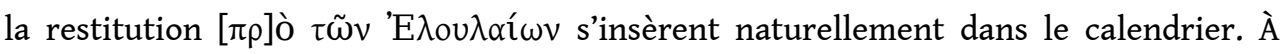

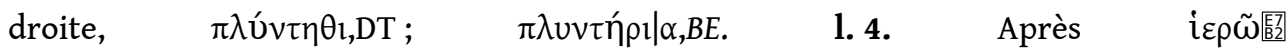

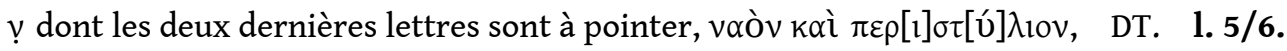

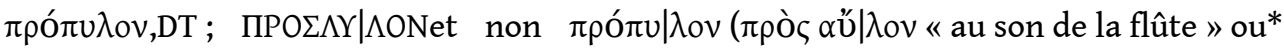

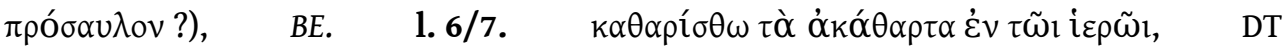

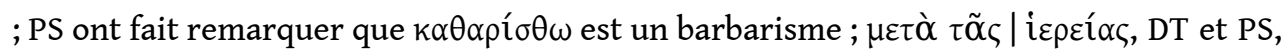
qui l'analysent comme une trace du dialecte thessalien; en fait, la voyelle de l'article se lit mal et il est inutile de supposer qu'il s'agit d'un A; en revanche, la forme

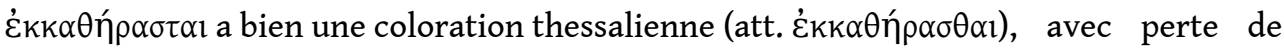
l'aspiration dans la désinence, comme pour la forme d'impératif

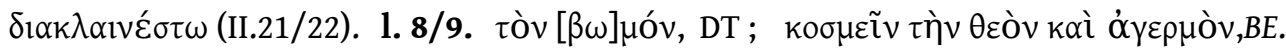
1. 9/10. Les lectures NEI $\left[\mathrm{F}_{2} \Pi \Lambda H N, D T\right.$, et NEIPE|!I $\Lambda \mathrm{HN}, B E$, ne se laissent pas plus

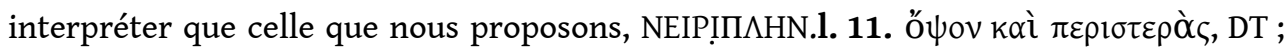

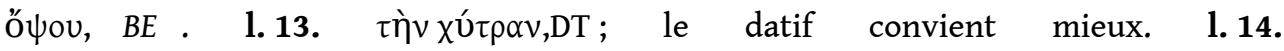

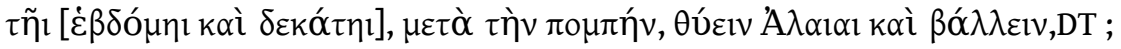

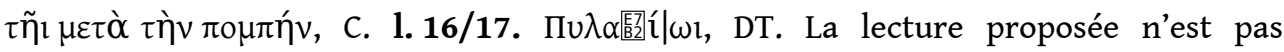
certaine: 1.16, lettre ronde puis haste qui, sur l'estampage et sous l'effet du charbonnage, suggère plutôt un upsilon. 1. 17. La première lettre est sans doute un chi;

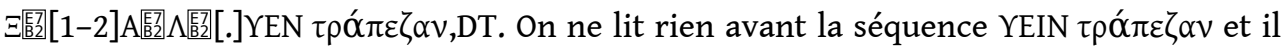

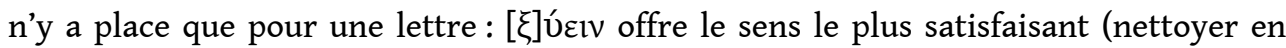

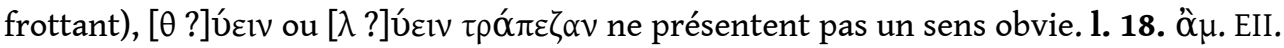

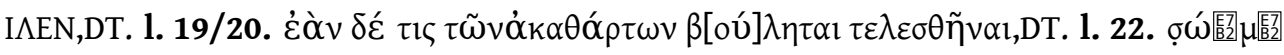
$\alpha$ 㹉 restituer $\dot{\alpha} \gamma \varepsilon[\rho] \mu о \tilde{,}, B E$, la première lettre de la 1.25 étant un $m u$, non lu par DT. 1. 25. $\pi \varepsilon \rho(\iota) \xi \cup \rho \varepsilon \tilde{\tau} \tau \alpha 1, \mathrm{DT} ; \quad \lambda \varepsilon i ́ \eta ı \pi \rho \circ \xi \cup \rho \varepsilon \tilde{\tau} \alpha 1, \quad B E, \quad 291$; $\quad$ DT ont oublié l'article

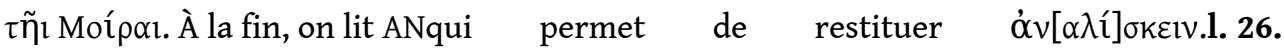

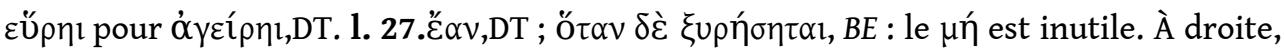

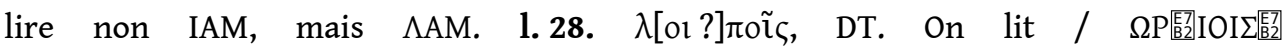
; la première lettre, sur l'estampage, présente une haste oblique accompagnée de deux points au dessus et en dessous, ce qui suggère, plutôt qu'un lambda, un chi incomplet, dont ne seraient conservées (gravées ?) qu'une haste et les extrémités de la seconde ;

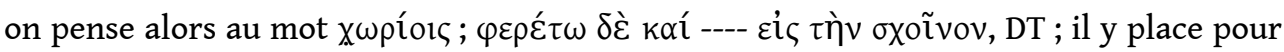

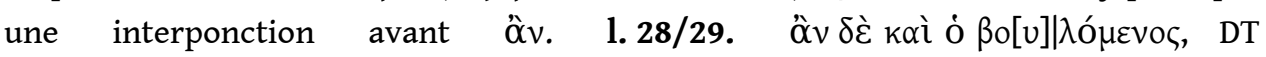
; les lettres conservées incitent à restituer $\widehat{\alpha} v \delta \varepsilon \dot{\varepsilon} \kappa \alpha \tau \alpha \beta[\varepsilon \mid \beta \imath \omega] \mu \varepsilon ́ v o \zeta$, mais on attendrait un participe avec le verbe $\kappa \alpha \tau \alpha \beta i \tilde{\omega}$. 1. 29. DT : après l'infinitif $\theta \varepsilon \rho \alpha \pi \varepsilon v ́ \varepsilon ı v$, la lettre qui suit n'est pas un sigma (DT), mais plutôt un nu ou un mu; plus loin on lit la fin d'un

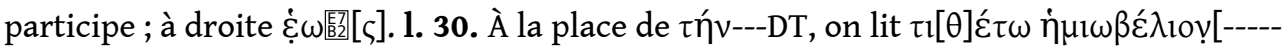

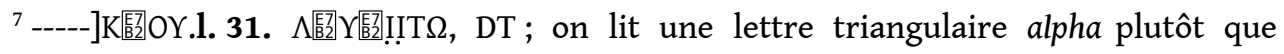

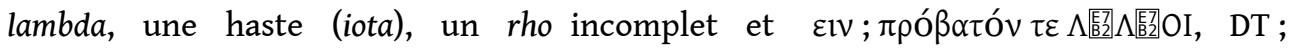

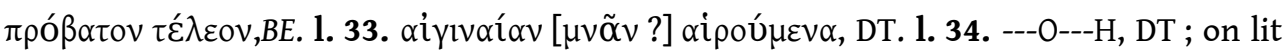

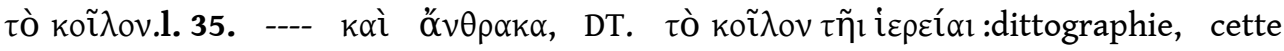

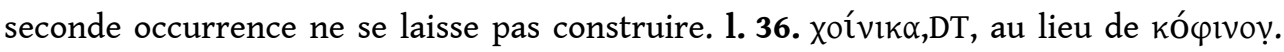
1. 37. $\tau \alpha(\tilde{v} \tau \alpha) \alpha \grave{u} \xi \alpha^{\prime} v \varepsilon 1 v \dot{\omega} \sigma \alpha u ́ \tau \omega \zeta, D T$. On ne sait à quoi correspond le conge, sans doute,

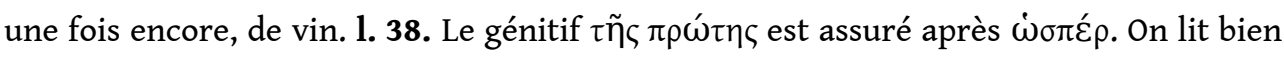

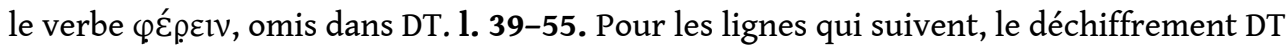


n'avait guère dépassé le stade de la copie en majuscules et la présente édition représente un progrès suffisamment important pour qu'on n'ait pas jugé utile de

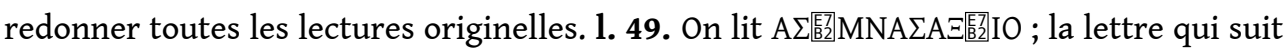
est soit un $\Sigma$ soit un T, mais l'objet de la $\theta v \sigma i ́ \alpha$ doit se rapporter à l'adjectif ö $\xi_{1 \circ \zeta}$ et donc être au masculin pluriel. On attendrait une coordination entre $\theta v \sigma \alpha ́ \tau \omega e t \tau \imath \theta \varepsilon ́ \tau \omega$, mais il ne semble pas y avoir assez de place en bout de ligne. 1. 50. La lacune qui suit le

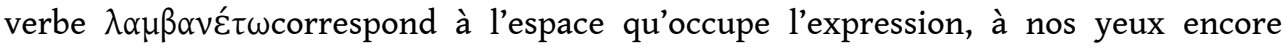

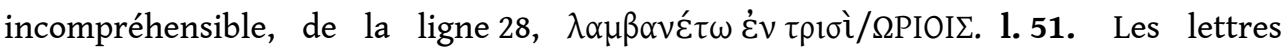

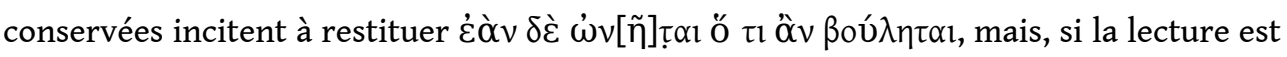

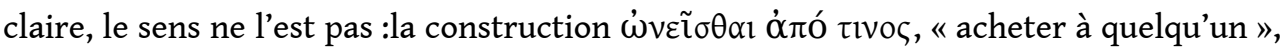

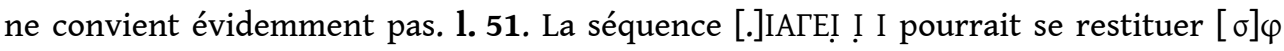

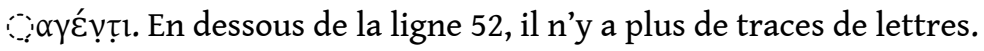

\section{Traduction}

\section{Face I}

5 Que, dans le temple de la déesse, aucun non-initié ne pénètre; si un non-initié entre, accomplir la purification avec une poule et sacrifier ensuite une seconde volaille adulte sur l'autel de Moire, ${ }^{4}$ et que procède à la purification soit la prêtresse, soit le néocore, soit l'une de celles qui portent les objets sacrés, et qu'on apporte deux chénices de pain et huit cotyles de vin pour le cratère.

6 - Dans le vestibule, qu'aucun non-initié ne pénètre, ${ }^{8}$ à moins de vouloir adresser des prières, et qu'il ait des vêtements purs et qu'il se garde pur pendant les trois jours, en se tenant à l'écart de toutes les souillures gravées ci-dessus (ou affichées ?). Qu'il apporte pour les prières un demi-cotyle d'huile pour la lampe, ${ }^{12}$ une obole, des torches, de l'encens et de quoi faire une libation.

7 - Si l'un des non-initiés pénètre dans le vestibule, qu'on accomplisse la purification avec un coq ou une poule, qu'on fasse ensuite le sacrifice d'une patte de l'animal de son choix, sauf d'un porc, ${ }^{16}$ (qu'on apporte) trois chénices attiques de beignets et un demiconge de vin.

8 - Faire la quête, le premier jour du mois Itônios, sur les aires; ne pas faire la quête dans les maisons ni y introduire les objets sacrés ; faire la quête du dix au douze, à moins qu'on ne fasse d'annonce trois jours à l'avance (?); si l'une des purificatrices n'accomplit pas cela, ${ }^{20}$ qu'elle paie comme amende au sanctuaire un mouton et le sacrifice y afférant.

9 - Inscription pour le péristyle. Qu'on fasse des offrandes en premier lieu à Phylakè et à Mèn, des offrandes d'encens.

10 - Si quelqu'un veut sacrifier des volailles blanches, ${ }^{24}$ qu'il sacrifie à Mèn des mâles et à Phylakè des femelles, et s'il veut sacrifier de jeunes agneaux, (qu'il agisse de) la même façon.

11 Pénétrer à l'intérieur (du sanctuaire) est autorisé à une accouchée au trentième jour, à celle qui a avorté au quarantième jour, à celle qui a eu commerce avec un homme, après s'être lavée ensuite de la tête aux pieds, à celle qui s'est purifiée de ce qui se produit ${ }^{28}$ selon la nature au septième jour. Si on entre sans s'être gardé desdites impuretés, qu'on purifie les autels avec un poussin, et qu'on offre en outre sur l'autel de Phylakè 
une poule ou un poisson apopyris, une mine de la viande qu'on veut, sauf de porc, ${ }^{32}$ une chénice de beignets et, pour le cratère, quatre cotyles de vin. Qu'accomplissent ces purifications le néocore et celle des purificatrices qui se trouve présente.

Si quelqu'un veut sacrifier à la déesse selon le rite grec, il est possible (de sacrifier) ce que l'on veut, sauf du porc. ${ }^{36}$ Pour ce sacrifice, il faut apporter sur la table les offrandes que l'on dépose: une chénice de beignets, une chénice de bouillie au sésame, une triobole pour le tronc, un cotyle d'huile pour la lampe et, pour le cratère, un conge de vin provenant du sanctuaire. (Déposer) sur la table la poitrine cuite et la patte crue ; pour la prêtresse, faire cuire les entrailles, le foie, ${ }^{40}$ le poumon, les viscères, le rein gauche et la langue ; le rein droit, le pied (?) droit, le cœur, la membrane intestinale, la patte détachée de la poitrine et de la queue ce qui est d'usage, (qu'on les apporte) pour offrandes sacrées sur le feu. Après avoir fait cela et après avoir accompli le sacrifice, qu'on apporte une autre victime de l'espèce que l'on veut ${ }^{44}$ et qu'en mange qui veut.

13 - Si quelqu'un veut faire table pleine où rien ne manque pour la déesse, (prendre) un ovin adulte, mâle ou femelle. Le faire cuire en entier; (apporter) deux chénices de bouillie au sésame, quatre chénices de beignets, fournir deux conges de vin, une drachme pour le tronc, un cotyle d'huile ${ }^{48}$ pour la lampe - et le non-initié n'en mange pas.

14 Si quelqu'un veut consacrer toute une journée à la déesse, (qu'il le fasse) en apportant comme déjeuner ce qu'il veut, sauf de la viande de porc, et pour la lampe un demicotyle d'huile.

15 - Pour l'oreille de la déesse et ses cheveux, de l'encens, de la myrrhe, ${ }^{52}$ du thymiama, des plantes aromatiques, de l'essence de rose, trois oboles pour le tronc.

16 - Si l'on sacrifie une volaille ou une oie: pour la volaille, qu'on verse au tronc une obole, pour l'oie, une obole et demie ; qu'on dépose les pattes et les viscères sur la table, le reste qu'on l'emporte où l'on veut ; qu'on apporte aussi ${ }^{56}$ quatre cotyles de vin, une chénice de beignets, un demi-cotyle d'huile.

17 - Si on veut sacrifier un bœuf, qu'on apporte sur la table trois chénices de beignets, une chénice de bouillie au sésame, deux conges de vin, deux cotyles d'huile, du bois en suffisance, pour le tronc une pièce d'or et qu'on prélève les parts sacrées comme dans le cas ${ }^{60}$ des ovins.

18 - Que tous ceux qui veulent sacrifier lors des Nisanaia ou des Aloulaia conduisent la victime à la procession. Que la procession pour les Nisanaia se tienne quand (?) la déesse arrive du fleuve, le lendemain; pour les Aloulaia, le 17 du mois au matin; à la nuit, qu'on fasse une procession aux flambeaux; que participe à la procession qui le veut, ${ }^{64}$ après qu'il s'est lavé le jour même de la tête au pied, et qu'il pénètre jusqu'au sanctuaire de Phylakè.

19 - Si quelqu'un veut faire l'holocauste d'un bélier adulte ou, à défaut, d'un agneau mâle, qu'il verse, pour le bélier, un statère au tronc et, pour l'agneau, une drachme éginétique, qu'il fournisse du bois en suffisance pour cela, un conge de vin pour le cratère, sur la table ${ }^{68}$ une chénice de bouillie au sésame, une chénice de beignets, de la rue des jardins et de la rue sauvage, de l'encens, de la myrrhe, un cotyle d'huile pour la lampe.

20 - Si l'on fait l'holocauste d'une oie, qu'on verse au tronc deux oboles, qu'on fournisse du bois en suffisance, une torche, quatre cotyles de vin pour le cratère, un demi-cotyle 
d'huile pour la lampe, de la rue des jardins et de la rue sauvage, de l'encens, ${ }^{72}$ de la myrrhe.

21 Si l'on fait l'holocauste d'un oiseau trybba ou d'une caille, qu'on verse une obole et demie au tronc; pour le reste, tout comme pour l'oie.

Que le non-initié ne s'approche pas du grand autel ; qu'on n'y sacrifie pas de volaille, ni n'y apporte d'œuf, mais, si l'on sacrifie à la déesse une volaille, (qu'on le fasse) sur l'autel de Moire ; ${ }^{76}$ et qu'accède qui le souhaite à l'autel de Moire et d'Hélios. Si un noninitié parvient jusqu'au grand autel, qu'on fasse la purification selon la procédure, exposée précédemment, de purification du péristyle.

Qu'on se tienne aussi de la même manière à l'écart de l'autel d'Adara et Lilla. Si quelqu'un les (= les autels) touche, qu'on accomplisse la purification selon l'affichage ${ }^{80}$ du péribole. Si quelqu'un, sous le péristyle, urine ou saigne, qu'on procède à la purification indiquée ci-dessus.

\section{Face II}

des

sacrifices,

d'abord-

le 5 Avant les Éloulaia, le 12. Qu'accomplisse un sacrifice préliminaire à Moire, collectivement ou à titre privé, celui des initiés ${ }^{4}$ qui le veut. Le 13. Lavage des objets sacrés (= vêtements ?) qui concernent la déesse, dans leur ensemble ---, et des tambourins et au son de la flûte, et sacrifice à Mogga et purification: que procèdent à la purification dans le sanctuaire, avec la prêtresse, les purificatrices, le néocore et tout autre initié qui le veut. ${ }^{8}$ - Le 14. Parer la déesse et consacrer le produit de la quête à Hélios. Le 15. Que sacrifie à Pan que les Syriens appellent NEIPIח $\Lambda \mathrm{H} \Sigma$ qui le veut et qui le désire ; qu'il mette sur la table ce qu'il veut, sauf poisson et colombe, et que celui qui fait le sacrifice place ${ }^{12}$ sur la table ce qu'il veut et qu'il prenne en échange ce qui est déposé sur la table. Puiser de l'eau aussi avec le pot de terre, eau puisée d'une fontaine, lors de la cérémonie nocturne. - Le 16. Ouvrir le pot de terre, et qu'offre un sacrifice à Moire qui le veut. Le lendemain de la procession, sacrifier à Adara et asperger avec du sel. ${ }^{16}$ Le 19. Asperger (?) avec du sel en

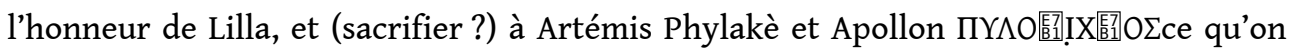
veut et que tous consomment. Le 20. Nettoyer (?) la table et faire un sacrifice (complémentaire ?) à la déesse de ce qu'on a.

Initiation (aux mystères) de la déesse. Dans le cas où quelqu'un est initié à sa demande, qu'il soit trois jours de service; le troisième jour, qu'il se rase. Dans le cas où c'est un des impurs qui veut être ${ }^{20}$ initié, qu'il se rase dans les trois jours ; qu'il se rase après avoir pris du vin; et que le pur de la déesse fasse le geste de laver (?) la bouche par l'or et par une plante et que l'impur en cours d'initiation fasse le geste de laver (?) la bouche avec le sang d'un oiseau, et que les purificatrices consomment ce dernier. Que celui qui est en cours d'initiation ne sacrifie pas, ${ }^{24}$ mais qu'il apporte une chénice attique de beignets et deux cotyles de vin de la quête; il faut qu'il fasse la quête le troisième jour, celui où il se rase, et qu'il sacrifie à Moire un oiseau et qu'il consomme le produit de la quête. Qu'il paie aussi à la déesse pour la corbeille avant d'être rasé, une triobole ; lorsqu'il est rasé, une obole d'offrande expiatoire ; qu'il prenne (la corbeille ?) ${ }^{28}$ dans trois (endroits ?) ; et qu'il apporte --- pour (?) la corbeille ; et si, passant sa vie à servir (?), ---- par la déesse, qu'il fasse son service tant qu'il est en bonne santé, et qu'il dépose une demi-obole ------ et le pain. 
Bassin. Quand on soulève le premier, soulever près de Moire et sacrifier du petit bétail adulte, ${ }^{32}$ mâle ou femelle selon ce qu'on veut, pur, (apporter) quatre chénices de beignets, deux conges de vin pour les mains, une drachme éginétique, les parts sacrées choisies comme pour la table pleine; séparer la patte non cuite et l'intestin pour la prêtresse et qu'elle les consomme ; cela et le reste, tous ceux qui sont non-initiés n'en mangent pas ; l'intestin pour la prêtresse. ${ }^{36}$ Pour qui soulève (?) le second bassin, un kophinos de beignets, une chénice de bouillie au sésame, un cotyle d'huile pour les mains, un statère pour le cratère, deux conges (de vin ?), prélever les parts sacrées comme celles du premier ; qu'on emporte où on veut, parmi les initiés ; asperger (?) de sel et sacrifice; les victimes 40 pour les mains, les corbeilles et si on (veut faire quelque chose ?) des pots de terre -------------------- le pot de terre se renverser ; si on veut ------ en songe ---- dans un pot de terre, ${ }^{44}$ sacrifier comme ovin à Hélios ce qu'on veut-------asperger (de sel ?)

${ }^{48}$ Serment. Si l'on prête serment --------, ayant pris comme équipement (?) de la déesse ce qu'on veut, qu'on sacrifie----- d'une valeur d'une mine(?); qu'on dépose une triobole et qu'on prenne ------ ; et si un non-initié ----- par (?) l'autel, qu'il boive ; s'il achète (?) ce qu'il veut par (?) le poisson, ${ }^{52}$ qu'il boive et qu'il dépose une drachme éginétique, et qu'il se garde pur aussi longtemps qu'il vit; si l'on fait un serment de moindre importance (?), après s'être lavé des pieds à la tête, qu'on prononce le serment à haute et intelligible voix et qu'on dépose une demi-obole pour la bête égorgée (?) ; à celui qui a égorgé et à qui a prélevé les parts sacrées, les demi-oboles.

\section{Commentaire}

\section{Ordre des faces et organisation du texte}

28 L'une des deux faces est bien plus usée que l'autre : l'épiderme en a disparu à de nombreux endroits, surtout dans la partie centrale, les bords ayant été mieux conservés et étant parfois remplis de concrétions calcaires. Les premières lignes déchiffrables se trouvent à $23,5 \mathrm{~cm}$ de la moulure supérieure de la stèle. La première édition du texte supposait prudemment que les premières lignes gravées sur cette face n'étaient plus lisibles: elles devaient alors former le début du texte et contenir toutes les informations qui manquaient pour bien le comprendre (autorité responsable de la gravure, nom de la divinité principale, description des fêtes mentionnées dans le reste du texte de manière allusive). Cependant, un examen minutieux des photographies et de la pierre elle-même fait douter de l'existence de ces lignes perdues. C'est probablement l'usure de l'eau qui a rendu la face difficile à déchiffrer par endroits, mais les zones proches des rebords gauche et droit ont conservé leur épiderme et aucune trace de lettres n'y est décelable.

Nous sommes donc d'avis qu'il ne faut pas exclure la possibilité qu'il ne nous manque pas grand-chose du texte complet inscrit. Le graveur a pu commencer son texte à plus de $20 \mathrm{~cm}$ du haut du champ disponible, tout comme il l'a interrompu bien avant le bas de la stèle, centrant ainsi son texte. Une telle disposition appuie l'hypothèse selon laquelle cette face usée est la seconde.

Il convient d'être prudent et nous avons rassemblé un certain nombre d'autres arguments qui vont dans le sens de cette hypothèse. 
31 Le premier argument en faveur de l'interversion de l'ordre des faces (A devenant II et B devenant I) repose sur la gravure - sans tenir compte, bien entendu, de la qualité de la préservation de chaque face. La première moitié de la face I a des lettres sensiblement plus grosses et plus espacées (en moyenne de 0,9 à $1 \mathrm{~cm}$, hormis les 0 ; environ 40 lettres par ligne) que dans la moitié inférieure et que tout ce qui est préservé de l'autre face (en moyenne $0,7 \mathrm{~cm}$ et de 50 à plus de 55 lettres par lignes). Pour autant, on ne remarque pas de changement de main dans la gravure et c'est assez progressivement que l'écriture se resserre. Les paragraphoi, même s'ils sont moins nombreux et moins réguliers à la fin de la face I, le sont nettement plus que sur la face II (nous n'en avons repéré que deux, II.8, 14), comme si cette dernière avait fait l'objet de moins d'attention de la part du graveur.

32 Second argument. Notre face I comporte un certain nombre de dispositions d'ordre général qui touchent l'ensemble des personnes susceptibles de s'approcher du sanctuaire. En particulier les non-initiés - ceux qui ne paraissent pas devoir s'inscrire dans le processus de l'initiation : ils sont là, tout au plus, pour prier - ne sont cités qu'ici, au tout début de cette face $(\mathrm{I} .1,8)$ et ne sont plus mentionnés. La face II traite en revanche de questions plus précises (préparation et déroulement de la fête; cérémonies des mystères) et donne une impression d'énumération avec ses trois

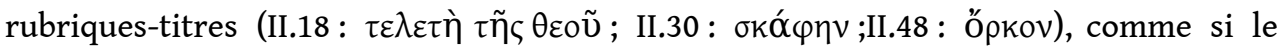
graveur avait rassemblé des informations venant de plusieurs dossiers d'archives ${ }^{5}$.

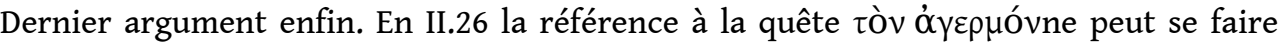
que par un renvoi à celle dont les modalités sont indiquées en I.17-19, de même que le renvoi aux modalités de la $\tau \rho \alpha \pi \varepsilon \zeta o \pi \lambda \eta \sigma i ́ \alpha$ en II.34-34 doit faire référence à ce qui est prescrit en I.44-48. Certes, les prescriptions de pureté imposées en I.8, dites $\pi \rho \bar{\varepsilon} \rho \alpha \mu \mu \varepsilon \varepsilon^{\prime} \alpha$, paraissent aller à l'encontre de notre hypothèse, mais il faut peut-être

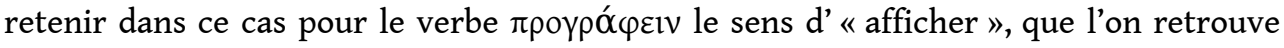

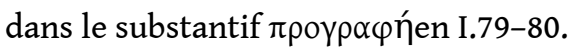

Cette interversion a, si nous avons raison, une conséquence immédiate. Le texte nous fait entrer directement in medias res et il paraît très difficile, voire impossible, de supposer qu'ait été mentionnée, dans le tout début de la face II, une quelconque autorité responsable de la gravure. Cela n'interdit évidemment pas pour autant de s'interroger sur l'identité de cette autorité, la cité sur le territoire de laquelle était installé le sanctuaire, ou l'association chargée du sanctuaire, comme le proposaient, sans trancher, J.-C. Decourt et A. Tziafalias ${ }^{6}$, ou un thiase analogue à celui des Alouliastai de Cos, selon l'hypothèse très stimulante de J.-M. Carbon ${ }^{7}$. R. Parker et S.Scullion, précisément parce qu'ils ne peuvent admettre que le texte entre immédiatement dans le vif du sujet, sans mention d'autorité et sans mention du nom de la déesse, refusent que l'inscription commence par la face I (face B pour eux) - sauf à supposer l'existence d'une autre stèle, présentée comme une hypothèse impossible à argumenter par Decourt et Tziafalias et qu'ils récusent eux-mêmes ${ }^{8}$.

\section{Calendrier}

J.-M. Carbon a rassemblé, dans un tableau très utile (p. 207), tous les éléments liés à la fête des É/Aloulaia mentionnés sur les deux faces de la stèle: les nouvelles lectures permettent de le compléter et d'amender quelques points de détail (date de début et fin des festivités ; nom des divinités successivement honorées). 

cultuel détaillant les fêtes sur un mois entier : nous estimons plutôt que toutes les dates évoquées concernent directement ou indirectement la fête des É/Aloulaia ${ }^{12}$. En effet, à la suite des prescriptions journalières de la fête des

É/Aloulaia (II.3-18), aucune mention de date n'apparaît plus (les doutes exprimés par J.M. Carbon, p. 188-189, sur les lectures de II.44 et 47 étaient justifiés). La fête à proprement parler se déroule du $13^{\mathrm{e}}$ au $20^{\mathrm{e}}$ jour; elle s'ouvre par une cérémonie de lavage des objets/vêtements sacrés et se clôt par le sacrifice à la divinité principale du sanctuaire; elle est précédée le $12^{\mathrm{e}}$ jour par un sacrifice préliminaire à Moire, dit

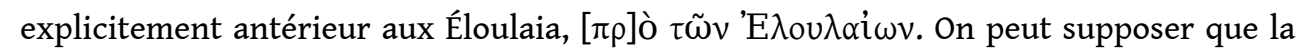
mention du $5^{\mathrm{e}}$ jour ( $\tau \tilde{\eta} 1 \pi \varepsilon \dot{\varepsilon} \mu \pi \tau[\eta 1]$ II.1) devait concerner une opération de préparation à la fête, à l'instar des quêtes (agermoi) évoquées sur l'autre face, qui avaient lieu le $1^{\mathrm{er}}$ jour du mois et pour une période de trois jours, à partir du 10 jusqu'à la veille du début des festivités. Les prescriptions ayant trait aux quêtes n'étaient probablement pas reprises sur la face II (anc. face A) - la lacune II.2 serait d'ailleurs bien trop courte pour les contenir -, pas plus que n'y a été reprise la mention de la procession le $17^{\mathrm{e}}$ jour. En revanche, il est tout sauf certain qu'on puisse placer chronologiquement les cérémonies

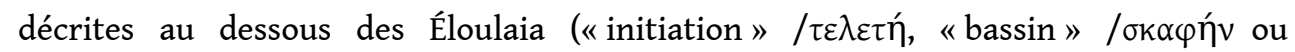
« serment "/Ǒpkov) par rapport à celles-ci. Pour autant, J.-M. Carbon a eu raison de faire remarquer que les cérémonies d'initiation auraient tout intérêt à avoir lieu avant la tenue des Éloulaia, qui restreignent la plupart des gestes et sacrifices aux seuls initiés : aussi la période de trois jours pour le service de la déesse et la quête le

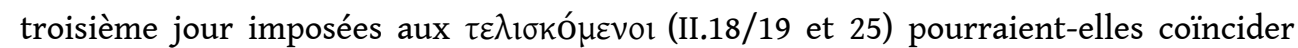
avec les trois jours liés à une proclamation officielle et à une quête (I.17-20) de la première face, qui devaient précéder la fête des Éloulaia. Cependant, le passage, qui a fait l'objet d'une correction par le lapicide, est tout sauf clair ${ }^{13}$.

\section{Divinités}

On ne reviendra pas en détail sur l'identité des différentes divinités ${ }^{14}$ dont les noms apparaissent dans ce document, mais quelques remarques s'imposent néanmoins. Un meilleur déchiffrage fait disparaître (II.15) la déesse Alaia et la déesse Lillaia (II.16).

Pour deux divinités dont les épiclèses n'étaient pas vraiment assurées, Pan (II.9) et Apollon (II.16), nous suggérons deux nouvelles lectures. Pour l'épiclèse de Pan, la

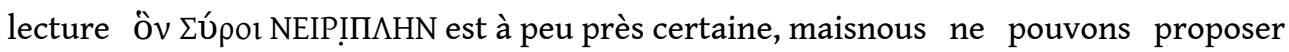
d'interprétation ni, par conséquent, de rapprochement avec une divinité orientale ${ }^{15}$.

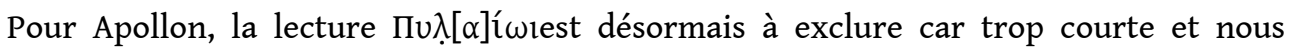

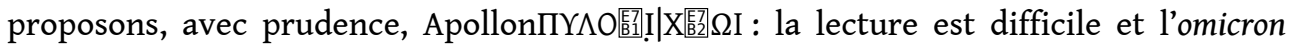
pourrait être un alpha et le chi, à la rigueur, un kappa. On serait tenté de restituer $\Pi \cup \lambda \alpha$ 


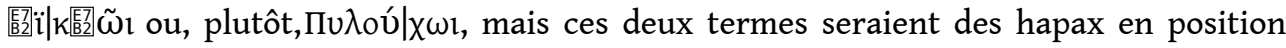
d'épithètes cultuelles ${ }^{16}$. Quoi qu'il en soit du libellé exact de l'épiclèse, l'association d'un Apollon des Portes et d'une Artémis Gardienne (II.16) apparaît naturelle.

Elle irait en tout cas bien dans le sens de l'hypothèse de R. Parker ${ }^{17}$, selon laquelle la titulaire du sanctuaire n'était pas Artémis Phylakè, mais une divinité certainement orientale et pour nous anonyme, que son nom ait été gravé dans la partie détériorée de la face I ou qu'il soit resté dissimulé aux non-initiés.

\section{Archéologie du sanctuaire}

Dans ce domaine aussi, nos relectures, ainsi que les remarques des commentateurs, nous conduisent à modifier, au moins partiellement, l'image qui avait été donnée du

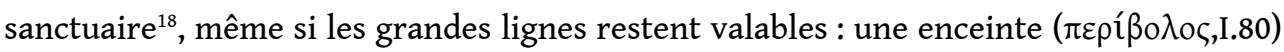
dotée d'un vestibule ( $\pi \rho o ́ \theta v \rho o v, ~ I .7,13)$ enclot plusieurs autels - mais le $\pi \varepsilon \rho[1] \sigma \tau[u ́] \lambda_{1 o v}$ repéré par Decourt et Tziafalias (I.4/5) doit disparaître. En revanche, le sanctuaire comportait bien un péristyle, $\pi \varepsilon p l \sigma \tau u ́ \lambda o v$ (I.21), qui portait gravée une inscription, mais dont l'emplacement dans la topographie du sanctuaire, n'est pas connu. De même

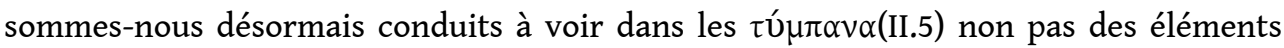
architecturaux, mais, plus banalement pourrait-on dire, des instruments de musique, associés à l'aulos dans le rituel ${ }^{19}$.

Les autels, à une exception près (II.51), ne sont mentionnés explicitement que sur la face I. Sont cités un autel de Phylakè, et un d'Adara et Lilla. Nous serions moins catégoriques sur l'existence de deux autels à Moire, l'un pour elle seule, l'autre où elle serait associée à Hélios $(\mathrm{I} .75,76)$, même si les deux mentions sont un peu différentes et suggèrent donc deux monuments distincts : le sacrifice est destiné à Moire seule, mais l'autel peut être double. Quant au grand autel, qui apparait plusieurs fois (I.73/74, 77), il l'est sans que jamais ne soit mentionnée sa titulaire, ce qui n'a rien d'anormal - il n'y a qu'un seul grand autel dans le sanctuaire, dont tous connaissent la titulaire - mais cela en conforte, pour nous, l'anonymat.

Quant aux installations annexes, deux points méritent d'être relevés. Nous faisons nôtre la remarque de J.-M. Carbon ${ }^{20}$ sur la valeur générique du terme oíkía (I.17), qui désigne non un bâtiment spécifique, mais un ensemble de maisons: on ne comprendrait pas bien, du reste, l'interdiction d'une quête dans une maison en particulier, sans autre précision sur le motif de cette interdiction. Sur les aires (

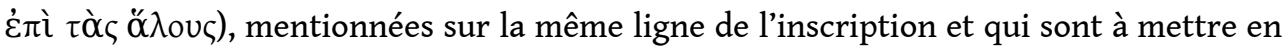
rapport avec l'oikía, voir supra.

\section{Personnel du sanctuaire}

L'organisation du personnel du sanctuaire peut désormais être précisée. Le culte de la déesse titulaire est assuré par une prêtresse seule. Cette dernière n'apparait que trois fois (I.39, II.7, II.34/5), comme bénéficiaire de parts des victimes qu'elle semble devoir manger sur place ou comme responsable de la purification du sanctuaire à l'occasion des Éloulaia.

À ses côtés intervient, outre le néocore (II.7), un collège dont les membres sont appelés purificatrices (II.7: $\tau \grave{\alpha} \varsigma \varphi \circ ß \beta \alpha \tau \rho i ́ \alpha \varsigma)$ ). Ces purificatrices sont apparemment chargées 
aussi de la quête (I.19/20), à moins que leur négligence éventuelle, pour laquelle elles peuvent être mises à l'amende, ne concerne que l'annonce de cette quête:

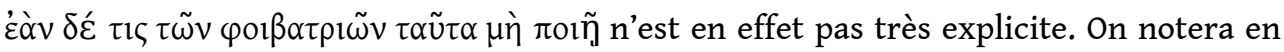
outre que l'initié peut participer à la purification du sanctuaire, qui n'est donc pas réservée à un personnel spécialisé (II.7/8) :

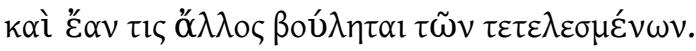

Ce sont des femmes et non des hommes qui accomplissent un geste sacré important (I.

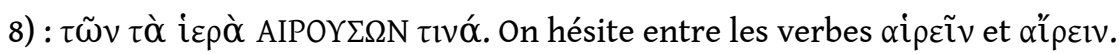
Les deux verbes ou leurs composés apparaissent dans l'inscription : aîpziven II.31, દ̇ò $v$

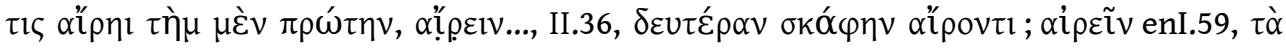

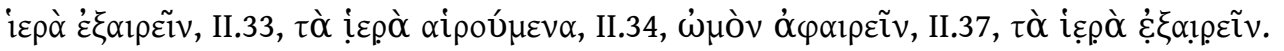
Dans le contexte de la phrase, il nous semble difficile de trancher résolument: le

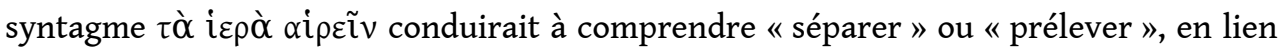
avec les parts sacrées, mais le fait que ce soient des femmes qui agissent nous pousse à préférer le sens de « soulever, porter ».

\section{Quelques remarques sur les rites}

48 - Le vin est, comme il est normal, mentionné à de nombreuses reprises, mais on peut s'arrêter sur quelques points. L'affirmation de Decourt et Tziafalias ${ }^{21}$ selon laquelle il y avait, dans le sanctuaire, une vigne, doit être prise avec prudence, dans la mesure où la

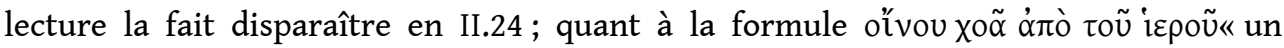
conge de vin provenant du sanctuaire » (I.38), elle est ambiguë et peut désigner soit du vin produit dans le sanctuaire, soit du vin qui y est stocké pour être revendu aux fidèles $^{22}$. Un autre passage irait dans le même sens (II.24): l'initié apporte

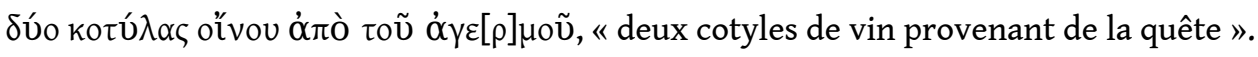

49 - La cérémonie pour l'oreille et les cheveux de la déesse devait donner lieu à des fumigations multiples : une deuxième sorte (qualité ?) d'encens, $\theta u \mu i ́ \alpha \mu \alpha$ (I.52/3), est utilisée en plus du $\lambda_{1} \beta \alpha{ } v \omega \tau o v(\mathrm{I} .12,22,68,71)$. Pour les holocaustes, le règlement

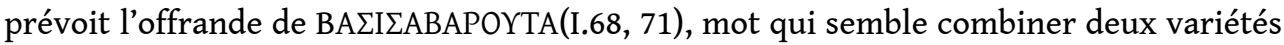
de la plante nommée «rue»: on y reconnait un premier membre sémitique (voir le syriaque bashasha), que le grec a adopté sous la forme $\beta \eta ́ \sigma \sigma \alpha \sigma \alpha ; R$. Parker et S. Scullion suggèrent à juste titre de reconnaître dans le second membre non le latinruta, mais le

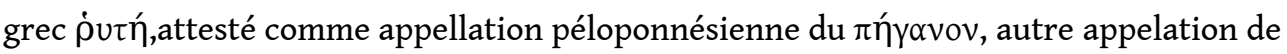
la rue ${ }^{23}$. Mais la forme même du mot gravé fait difficulté et nous avons abandonné la

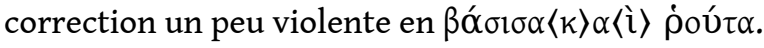

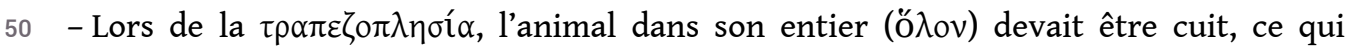
signifie qu'il n'était prévu de n'en prélever aucune portion, ni comme part sacrée, ni pour la prêtresse. La table est donc surtout pleine pour les mortels. L'action prévue est portée par le verbe ع̌ $\psi \varepsilon ı v$, dont le sens le plus fréquent, «bouillir », n'est satisfaisant pour aucune de ses occurrences dans le texte $(\mathrm{I} .38,39,46)$ : nous avons donc choisi de le traduire par un terme neutre « cuire».

51 - Le sens du verbe $\beta \alpha$ ó $\lambda \lambda \varepsilon ı v$ avait fait difficulté pour les premiers éditeurs et Parker et Scullion en avaient proposé plusieurs interprétation ${ }^{24}$. Il n'apparaît que sur la face II, à quatre reprises, et systématiquement précédé du complément ḋ $\lambda i ́$ (la dernière attestation [II.44] en restitution très probable, mais dans un passage en mauvais état). 
Le geste concerne le sel (au datif) ${ }^{25}$, que l'on jette, que l'on disperse. Dans deux cas, il est

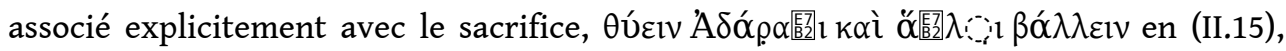

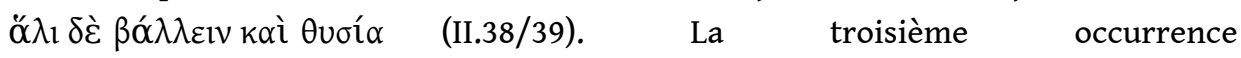

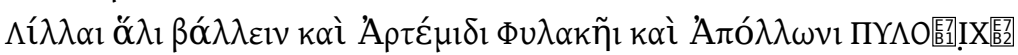

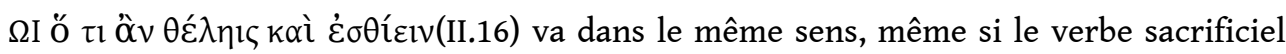
doit être sous-entendu. Ce geste de l'aspersion ${ }^{26}$ est donc associé étroitement au sacrifice, en l'honneur de divinités secondaires surtout (II.15, 16), mais aussi, même si on ne peut en être certain, en l'honneur de la divinité titulaire (II.38/39). Reste que cet usage du sel reste mystérieux - est-il utilisé seul, ou mêlé à de l'eau? -, même si, comme le remarquaient Parker et Scullion, son utilisation comme moyen de purification n'a rien de très étonnant.

52 - La quête (’’ $\gamma \varepsilon \rho \mu o ́ \varsigma)$ est mentionnée à trois reprises sur la face II, tandis qu'il est

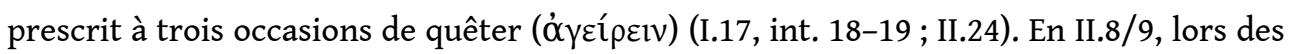

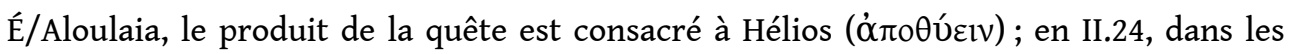
prescriptions liées à la $\tau \varepsilon \lambda \varepsilon \tau \eta ́$, l'initié apporte du vin pris « sur le produit de la quête » (

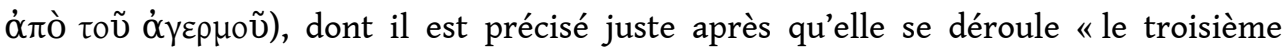
jour », au cours du processus d'initiation : il est tentant de rapprocher cette quête du troisième jour des trois jours de quête prévus les 10-12 Itônios ${ }^{27}$.

53 - Pour les Nisanaia, Parker et Scullion, qui rapprochent ce rite d'un autre mentionné

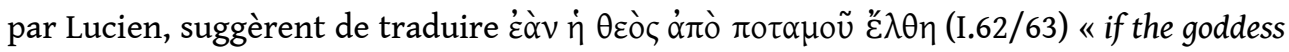
comes from the river", alors que nous préférons, comme Decourt et Tziafalias, garder le sens temporel, toujours possible, de la conjonction dans la traduction ${ }^{28}$. Mettre l'accent sur le caractère hypothétique du retour de la déesse a pour conséquence de mettre en doute la régularité annuelle du rite ${ }^{29}$.

54 - Parmi les objets qui sont mentionnés soit pour le culte, soit dans le cadre de l'initiation, figurent, outre la table nécessaire au sacrifice, plusieurs fois citée, les tambourins et l'aulos (II.5), un objet en jonc tressé, бxoĩvoৎ (II.26, 28, 42), peut-être une corbeille, un récipient en terre cuite, $\chi \dot{v} \tau \rho \alpha$, qui apparaît à plusieurs reprises, lors des Éloulaia, mais aussi dans des cérémonies dont le sens nous échappe malheureusement (II.13, 14, 42, 43), et enfin la øкó $\varphi \eta$ (II.30, 36). Ce dernier objet ${ }^{30}$, dont sont signalés deux exemplaires, est d'une particulière importance. Le terme recouvre en réalité toute une série de vaisseaux assez différents, mobiles, généralement en métal, et suffisamment

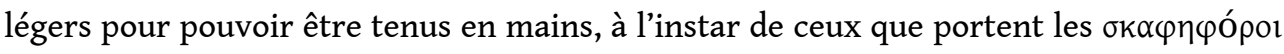
des Panathénées, dont les lexicographes nous apprennent qu'ils contenaient des gâteaux et des cierges et qui ressemblent somme toute à des plateaux. La traduction bowl de Parker et Scullion ${ }^{31}$ pourrait convenir, à condition d'y voir une coupe plus grande que le simple vase à boire et dans laquelle on peut déposer autre chose que des

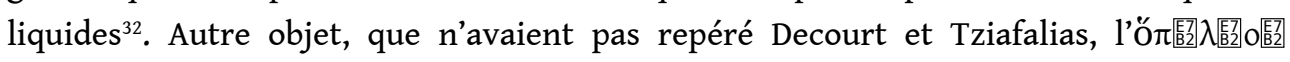
$v \tau \tilde{\eta} \varsigma \theta \varepsilon \circ \tilde{v}$ (I.49) dont, si les lettres sont pointées, la lecture est sure. Nous traduisons par un terme général « instrument », sens premier du mot, car rien, dans ce texte, ne permet de penser que la déesse revêt un aspect guerrier. On mettra enfin, au nombre

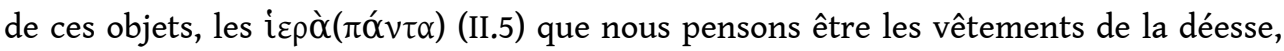
comme nous l'expliquons ci-dessous.

55 - Les actes de purification sont nombreux, dans le culte au quotidien, lors des fêtes ou des cérémonies d'initiation. Un terme attire l'attention, celui de $\pi \lambda u v \tau \eta ́ p l \alpha ~(I I .4 / 5)$ que nous traduisons par « lavage » et qui est un quasi hapax, puisqu'il n'est connu que par 
le nom de la fête athénienne des Plyntéria, célébrée le 25 Thargélion. Étant donné le

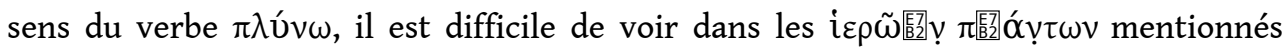
autre chose que des vêtements : est en cause la garde-robe de la déesse.

- Pour la cérémonie d'initiation proprement dite, un terme revient à trois reprises, celui de $\theta \varepsilon \rho \alpha \pi \varepsilon v ́ \varepsilon i v(I I .19,29)$. Nous le comprenons dans un sens religieux, « accomplir le

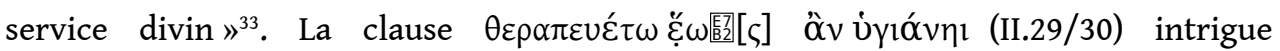

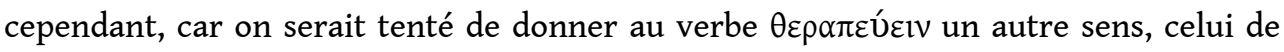

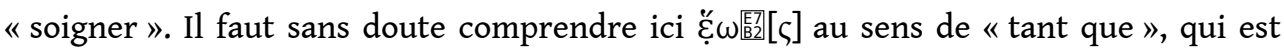

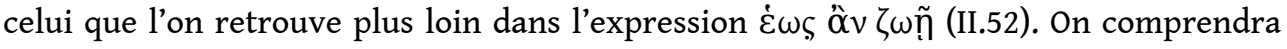
que le service divin peut ou doit être assuré par un individu " tant », ou " pour autant qu'il est en bonne santé ». Il est possible que cette dernière prescription ne concerne plus directement l'initiation, mais relève d'un paragraphe isolé ${ }^{34}$ : le texte semble envisager le cas d'un service permanent de la déesse (verbe $\kappa \alpha \tau \alpha \beta i \tilde{\omega}$, participe au parfait?, pour signifier qu'on veut finir ses jours, passer le restant de ses jours au service de la déesse), mais la phrase est incomplètement conservée et la syntaxe ne se laisse pas entièrement reconstruire.

57 - On doit à R. Parker et S. Scullion ${ }^{35}$ des remarques tout à fait stimulantes sur la cérémonie mésopotamienne du "Lavage de la bouche ", qu'ils mettent en rapport avec

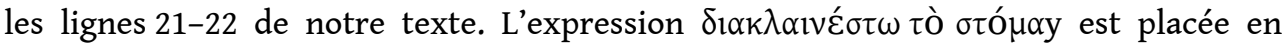
facteur commun pour exposer les modalités d'accomplissement de ce geste sacré par le pur ( $\mu \varepsilon ́ v)$ et l'impur $(\delta \varepsilon ́)$, mais le rédacteur semble avoir choisi de clarifier la deuxième partie de phrase en reprenant le groupe verbal qu'il a inséré au sein du groupe nominal $\alpha$ ¿̌́ $\mu \alpha \imath$ ópvı $\theta$ o $^{36}$. On comprendra, comme Parker et Scullion, et bien que l'expression

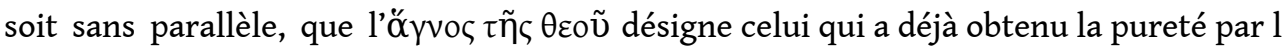

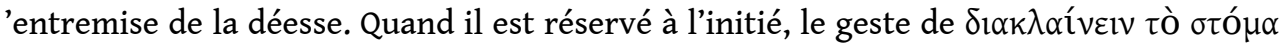
se fait avec des objets non sanglants (un objet en or, quel qu'il soit, et une plante, $\dot{\alpha}$

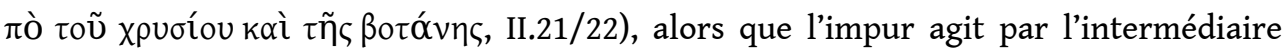
du sang de l'offrande d'un oiseau, tué pour être mangé par les prêtresses purificatrices (II.23/24).

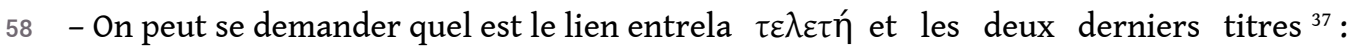
s'agit-il de moments de l'initiation ou, plus probablement, d'opérations entièrement indépendantes?

59 Ce qui touche au serment clôt notre inscription et cette partie est malheureusement en mauvais état de conservation. Il semble malgré tout que sont envisagées plusieurs catégories de serments ( 2 ou 3 ? Plus ? ${ }^{38}$ ), dans lesquelles seraient insérées des

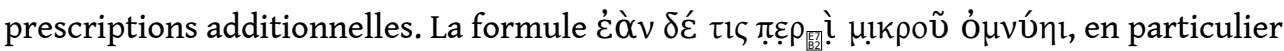
(II.52/53), pose problème : nous la traduisons avec prudence « si l'on fait un serment de moindre importance »: elle pourrait trouver un parallèle avec la toute première

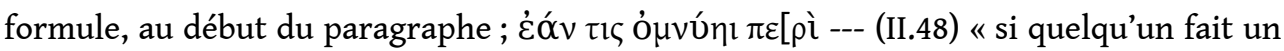
serment (sur un sujet important?) », la prestation de serment comprenant dans les deux cas le versement d'une somme d'argent (demi-obole, triobole) et un sacrifice.

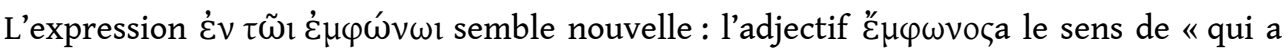

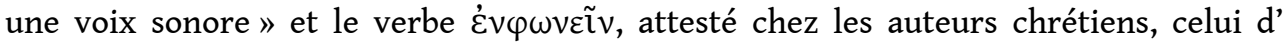
« appeler en criant ». 


\section{En guise de conclusion}

60 Le but du présent article était d'établir aussi prudemment que possible le long texte gravé sur la stèle de Marmarini qui est devenue à présent difficile d'accès, puisqu'elle fait partie de l'exposition permanente du Musée diachronique de Larissa. Les lectures et les hypothèses d'interprétation que nous avançons auront, nous l'espérons, fait progresser notre appréhension d'un texte difficile et qui ouvre des perspectives tout à fait nouvelles sur l'importance des cultes orientaux en Grèce continentale et leurs liens avec les cultes "indigènes ». Elles n'en épuisent évidemment pas la richesse et nombreux seront les chercheurs, nous n'en doutons pas, qui contribueront à améliorer encore la compréhension de ce règlement. 


\section{BIBLIOGRAPHIE}

J.-M. CARBON, « The Festival of the Aloulaia, and the Association of the Aloulaiastai: Notes Concerning the New Inscription from Larisa/Marmarini », Kernos 29 (2016), p. 185-208.

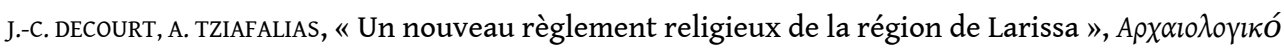

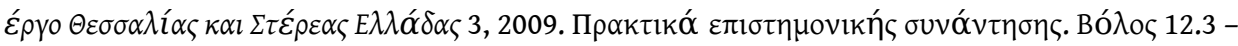
15.3.2009 (Proceedings of the 3rd Archaeological Meeting of Thessaly and Central Greece [AETHSE]), I, Volos, Ergastērio Archaiologias Panepistēmiou Thessalias, 2012, p. 463-473.

J.-C. DECOURT, A. TZIAFALIAS, « Un règlement religieux de la région de Larissa : cultes grecs et orientaux », Kernos 28 (2015), p. 13-51.

K. GALLIS, « Corn Transport from Thessaly to Rome in the 2nd c. b.C.: a Decree of the Thessalian League », ADelt 31 (1976 [1984]), p. 210-212.

P. GARNSEY, T. GALLANT, D. RATHBONE, « Thessaly and the Grain Supply of Rome », JRS 74 (1984), p. 30 44.

Br. HELLY, « Gloses thessaliennes et realia », in G. RocCA (ed.), Dialetti, dialettismi, generi letterari e funzioni sociali. Atti del V Colloquio di Linguistica Greca (Milano, 12-13 settembre 2002), Milan, 2004, p. 265-301.

R. HURSCHMANN, s. v. «Skaphe », Brill's New Pauly on line, 2006. 
V. MISAÏLIDOU-DESPOTIDOU, «A Hellenistic Inscription from Skotoussa (Thessaly) and the

Fortifications of the City », ABSA 88 (1993), p. 188-217.

R. PARKER, « The Nameless Goddess of Marmarini », ZPE 199 (2016), p. 58-59.

R. PARKER, S. SCULLION, « The Mysteries of the Goddess of Marmariani », Kernos 29 (2016), p. 209-266.

C. TRÜMPY, Untersuchungen zu den altgriechischen Monatsnamen und den Monatsfolgen, Heidelberg, 1997.

Fig. 1. Inscription de Marmarini, face I haut (lignes 1 à 25).

Nous remercions l'Éphorie des Antiquités préhistoriques et classiques de Larissa et sa Directrice, Madame Stavroula Sdrolia, de nous avoir permis de reproduire ces clichés.

Musée diachronique de Larissa, $15^{\mathrm{e}}$ Éphorie des Antiquités.

Fig. 2. Inscription de Marmarini, face I milieu (lignes 23 à 53).

Musée diachronique de Larissa, $15^{\mathrm{e}}$ Éphorie des Antiquités.

Fig. 3. Inscription de Marmarini, face I bas (lignes 53 à 81).

Musée diachronique de Larissa, $15^{\mathrm{e}}$ Éphorie des Antiquités.

Fig. 4. Inscription de Marmarini, face II haut (lignes 1 à 29).

HiSoMA-Archives thessaliennes de Lyon, Richard Bouchon.

Fig. 5. Inscription de Marmarini, face II milieu (lignes 19 à 33).

Musée diachronique de Larissa, $15^{\mathrm{e}}$ Éphorie des Antiquités.

Fig. 6. Inscription de Marmarini, face II bas (lignes 31 à 54).

HiSoMA-Archives thessaliennes de Lyon, Richard Bouchon.

\section{NOTES}

1. DECOURT - TZIAFALIAS (2015).

2. PARKER - SCULLION (2016), p. 265.

3. $B E$ (2016), 291-292-293; BE (2017), sous presse.

4. Il ne nous a pas paru possible, dans le cadre que nous nous sommes donné, de discuter en détail de toutes les propositions et hypothèses qui ont déjà été avancées, souvent de manière très détaillée, par J.-M. Carbon, R. Parker et S. Scullion, dont certaines, du reste, perdent de leur pertinence au regard du nouvel état du texte.

5. Le changement d'ordre des deux faces a pour conséquence d'isoler les mentions du terme

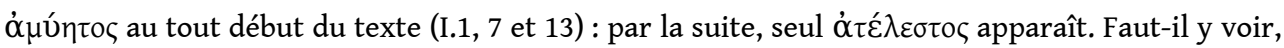
plutôt qu'une référence à deux degrés d'initiation, un signe du caractère composite du texte gravé, qui reprendrait des prescriptions qui ne furent pas élaborées dans un seul temps?

6. DECOURT - TZIAFALIAS (2015), p. 45-46.

7. CARBON (2016), p. 198-203.

8. DECOURT - TZIAFALIAS (2015), p. 45 ; PARKER - SCULLION (2016), p. 210.

9. Voir, vers $130 \mathrm{av}$. J.-C., le décret du koinon thessalien mettant en place la livraison à Rome de tout le blé disponible, sur requête de l'édile Q. Caecilius Metellus :Gallis (1976), avec photo pl. 127 (d'où SEG 34, 558, 1. 16-56); GARNSEY - GALLANT - RATHBONE (1984). Sous peine d'amende, les cités thessaliennes doivent acheminer vers les ports, au plus vite, les reliquats de l'année précédente 
et, au début du mois de Phyllikos, le produit de la nouvelle moisson: Phyllikos est le mois qui précède Itônios. Le battage a lieu vers le mois de juin.

10. HELLY (2004), p. 266-280, avance l'hypothèse selon laquelle la signification originelle du thème

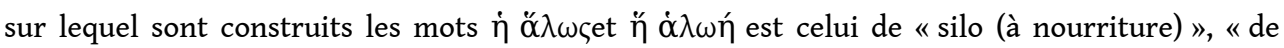
construction circulaire (au moins à l'origine) au-dessus du sol pour le stockage et la conservation des produits ». La fête athénienne des Halôia, qui a lieu pendant le mois hivernal Posidéôn, célébrerait «la protection et la sauvegarde des silos à blé, des greniers et des réserves de nourriture de toute sorte ». Un substantif $\dot{\alpha}$ *ö̀ovocest apparu dans une inscription de la cité

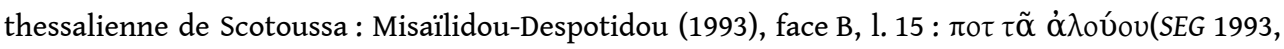
$311)$; les $\ddot{\alpha} \lambda \omega \varsigma$ du règlement sacré désignent peut-être des zones de stockage.

11. TRÜMPY (1997), p. 216-218, ignore l'évolution du calendrier thessalien, tel qu'il apparait dans la documentation épigraphique, et hésite inutilement sur la place des mois Hermaios et Apollônios.

12. Il n'est d'ailleurs pas impossible que seule la fête des É/Aloulaia ait donné lieu à une description complète: elle impliquait l'ensemble des neuf divinités associées au sanctuaire (Moire, Mogga, Lilla, Adara, Hélios, Pan NEIRIPLÈS, Artémis Phylakè, Apollon Pylouchos [?] et la déesse principale).

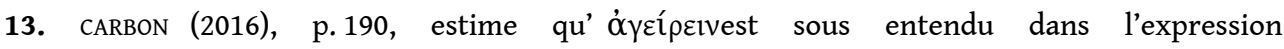

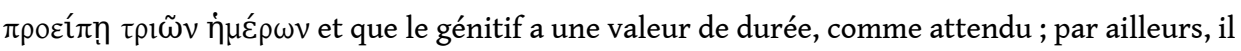

semble comprendre les deux propositions hypothétiques en I.18-19

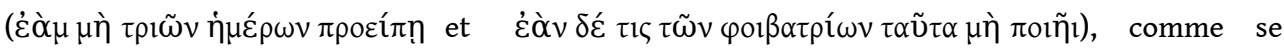
rapportant au même fait: les phoibatriai seraient soumises à amende "si elles ne font pas l'annonce que la quête dure trois jours ", "si elles ne font pas cela ». Si cette interprétation offre un sens cohérent, on est étonné que le lapicide n'ait pas choisi de faire disparaître par rasura les passages devenus inutiles. Il semblerait plus conforme à l'usage de considérer que le passage ajouté dans l'interligne est un passage qui avait été omis et que l'ensemble forme un tout

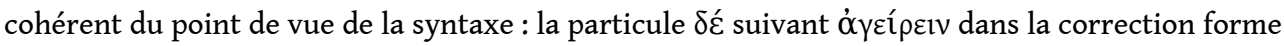
une articulation avec l'interdiction de quêter dans les maisons; la seconde particule $\delta \varepsilon ́$ ouvre la clause prévoyant l'amende pour les phoibatriai; la proposition subordonnée

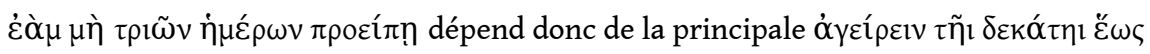

$\delta \omega \delta \varepsilon \kappa o ́$ in . PARKER - SCULLION (2016), p. 214, note 31, se demandent même si la prescription de quêter du 10 au 12 ne vient pas corriger la date proposée pour la quête. Ils comprennent

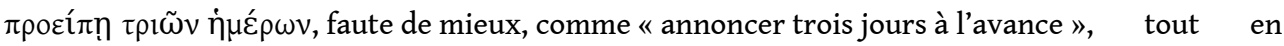
reconnaissant l'usage indû du génitif. Le passage reste donc embrouillé.

14. Voir les remarques complémentaires de PARKER - SCULLION (2016), p. 217.

15. Voir les suggestions de PARKER - SCULLION (2016), p. 217-218

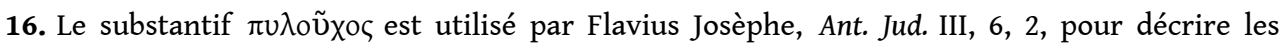
montants qui soutiennent les portes du tabernacle que Moïse fait dresser dans le désert en guise de temple. $Y$ a-t-il derrière $\pi \cup \lambda \circ \tilde{x} \chi 0 \varsigma$ un mot sémitique qui expliquerait aussi l'épiclèse $d$ 'Apollon?

17. PARKER (2015), et PARKER - SCULLION (2016), p. 217.

18. DECOURT - TZIAFALIAS (2015), p. 23-25 ; PARKER - SCULLION (2016), p. 218-220.

19. Hypothèse déjà avancée par CARBON (2016), p. 197, note 25 : kettledrums, « cymbales ", utilisées dans le culte de la Grande Mère ; repris par PARKER - SCULLION (2016), p. 213, note 18.

20. CARBON (2016), p. 198, note 27.

21. DECOURT - TZIAFALIAS (2015), p. 41.

22. Voir aussi sur ce point la remarque de PARKER - SCULLION (2016), p. 250-251.

23. PARKER - SCULLION (2016), p. 216, note 41. 
24. DECOURT - TZIAFALIAS (2015), p. 30-31; PARKER - SCULLION (2016), p. 254-255.

25. Le mot ö $\lambda$ ৎ a deux sens : au féminin, la mer, et au masculin, le sel, mais il ne désigne pas l'eau

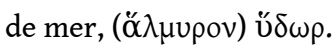

26. PARKER - SCULLION (2016), p. 254 suggèrent dans le premier cas pour ou sprinkle. Plutôt qu' " asperger », on pourrait aussi traduire « saupoudrer » : dans le premier cas, le sel est mêlé à de l 'eau (ou tout autre liquide), dans le second il est employé seul.

27. Voir CARBON (2016), p. 190 et supra le paragraphe sur le calendrier.

28. DECOURT - TZIAFALIAS (2015), p. 22 ; PARKER - SCULLION (2016), p. 216 et 229, repris par CARBON (2016), p. 196.

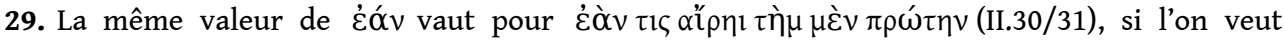
conserver le parallélisme avec ce qui suit pour le second bassin.

30. HURSCHMANN (2006).

31. PARKER - SCULLION (2016), p. 214.

32. Le terme apparaît chez Athénée, VIII, 2335 b, à propos du culte de la déesse Brizô à Délos : on

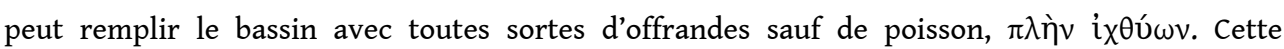
exclusion s'explique car il s'agit d'une déesse marine, mais on peut se demander si la même exclusion (II.11) dans notre texte est pure coïncidence. Le poisson apparait aussi dans une prescription que nous ne comprenons pas (II.51).

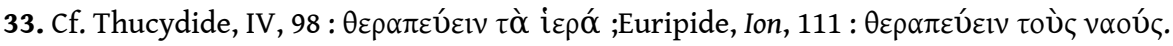

34. Voir les notes critiques.

35. PARKER - SCULLION (2016), p. 229-230.

36. Parker et Scullion ont bien souligné le problème soulevé par la syntaxe de la phrase qui implique nécessairement la présence d'une hyperbate soit, comme le comprenait Decourt et

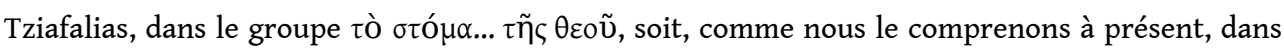

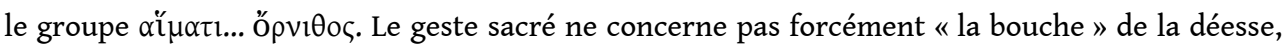
donc sa statue, ni, de façon parallèle, le « bec d'un oiseau ».

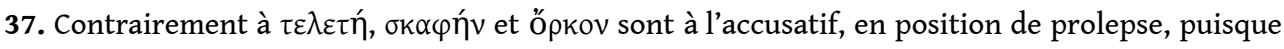
l'un et l'autre peuvent former l'objet du premier verbe de chacun des paragraphes qu'ils commencent.

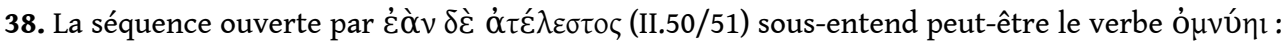

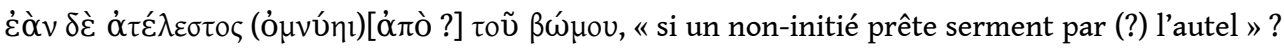
Il pourrait tout autant s'agir d'une clause additionnelle, emboîtée dans la liste des types de serments possibles. La prescription qui suit (II.51/52), impliquant peut-être un acte d'achat, n'est pas claire, mais elle est peut-être liée au serment par la notion de contrat?

\section{RÉSUMÉS}

Le long règlement religieux trouvé à Marmarini, au nord-est de Larissa (Thessalie), a déjà donné lieu, depuis sa publication, à plusieurs articles qui tentent d'éclairer ce texte difficile. Dans la présente contribution, les auteurs proposent de nouvelles lectures et une nouvelle traduction, ce qui les conduit à suggérer de nouvelles interprétations sur certains aspects du rituel de ce sanctuaire consacré à une divinité orientale qui n'est jamais nommée. 
The long religious regulation found in Marmarini, northeast of Larissa (Thessaly), has already given rise to several articles that attempt to shed light on this very difficult text. In the present contribution, the authors propose new readings and a new translation, which lead them to suggest new interpretations on some aspects of the ritual(s) of this sanctuary devoted to an Eastern divinity which is never named.

\section{AUTEURS}

\section{RICHARD BOUCHON}

UMR 5189 HiSoMA - Université Lumière Lyon 2

Maison de l'Orient et de la Méditerranée Jean-Pouilloux, Lyon

Richard.Bouchon@mom.fr

JEAN-CLAUDE DECOURT

UMR 5189 HiSoMA - CNRS

Maison de l'Orient et de la Méditerranée Jean-Pouilloux, Lyon

jean-claude.decourt@mom.fr 